\title{
Metallo-dielectric diamond and zinc-blende photonic crystals
}

\author{
Alexander Moroz* \\ Soft Condensed Matter, Debye Institute, Utrecht University, Postbus 80000, 3508 TA Utrecht, The Netherlands
}

(Received 18 March 2002; revised manuscript received 17 June 2002; published 11 September 2002)

\begin{abstract}
Diamond and zinc-blende photonic crystals are studied both in the purely dielectric case and in the presence of small inclusions of a low absorbing metal. It is shown that small metal inclusions can have a dramatic effect on the photonic band structure. Several complete photonic band gaps (CPBG's) can open in the spectrum, between the second and third, fifth and sixth, and eighth and ninth bands. Unlike in the purely dielectric case, in the presence of small inclusions of a low absorbing metal the largest CPBG for a moderate dielectric constant $(\varepsilon \leqslant 10)$ turns out to be the second to third CPBG. The second to third CPBG is the most important $\mathrm{CPBG}$, because it is the most stable against disorder. For a diamond and zinc-blende structure of nonoverlapping dielectric and metallo-dielectric spheres, a CPBG begins to decrease with an increasing dielectric contrast roughly at the point where another CPBG starts to open-a kind of gap competition. A CPBG can even shrink to zero when the dielectric contrast increases further. Metal inclusions have the biggest effect for the dielectric constant $\varepsilon \in[2,12]$, which is a typical dielectric constant at near infrared and in the visible for many materials, including semiconductors and polymers. It is shown that one can create a sizeable and robust second to third CPBG at near-infrared and visible wavelengths even for a photonic crystal which is composed of more than $97 \%$ low refractive index materials ( $n \leqslant 1.45$, i.e., that of silica glass or a polymer). In the case of silica spheres with a silver core, the second to third CPBG opens for a metal volume fraction $f_{m} \approx 1.1 \%$ and has a gap width to midgap frequency ratio of $5 \%$ for $f_{m} \approx 2.5 \%$. Within the second to third CPBG of $5 \%$, absorption remains very small $(\leqslant 2.6 \%$ once the CPBG is centered at a wavelength $\lambda \geqslant 750 \mathrm{~nm}$ ), which should be tolerable in most practical applications. The metallo-dielectric structures display a scalinglike behavior, which makes it possible to scale the CPBG from microwaves down to the ultraviolet wavelengths. Aluminum, copper, and gold cores yield almost identical results, provided that sphere radius $r_{s} \geqslant 250 \mathrm{~nm}$. For $r_{s}<250 \mathrm{~nm}$ the results for different metals can be increasingly different with decreasing $r_{s}$, nevertheless, qualitative features remain the same. These findings open the door for any semiconductor and polymer material to be used as genuine building blocks for the creation of photonic crystals with a CPBG and significantly increase the possibilities for experimentalists to realize a sizeable and robust $\mathrm{CPBG}$ in the near infrared and in the visible. One possibility is a construction method using optical tweezers, which is analyzed here.
\end{abstract}

DOI: 10.1103/PhysRevB.66.115109

PACS number(s): 42.70.Qs, 78.67.-n, 82.70.Dd, 87.80.Cc

\section{INTRODUCTION}

Photonic crystals are structures with a periodically modulated dielectric constant. In analogy to the case of an electron moving in a periodic potential, light propagating in a photonic crystal experiences multiple scattering leading to the formation of Bloch waves and photonic band gaps. ${ }^{1-3}$ If the band gap persists for both polarizations and all directions of propagation, one speaks of a complete photonic band gap (CPBG). ${ }^{4,5}$ Light with frequencies within a CPBG is totally reflected since it cannot propagate inside the crystal. Yet light can propagate through waveguides carefully designed within such a photonic crystal, even when such a waveguide has sharp bends. ${ }^{6}$ In the last decade, photonic crystals have enjoyed a lot of interest in connection with their possibilities to guide light and to become a platform for the fabrication of photonic integrated circuits. ${ }^{3,6}$ There is a common belief that, in the near future, photonic crystals systems will allow us to perform many functions with light that ordinary crystals do with electrons. In addition to numerous potential technological applications (filters, optical switches, superprisms, cavities, etc. ${ }^{3}$ ), photonic crystals also promise to become a laboratory for testing fundamental processes involving interactions of radiation with matter under novel conditions. ${ }^{1,2}$ The presence of a CPBG causes dramatic changes in the local density of states, which offers the possibility to control and engineer the spontaneous emission of embedded atoms and molecules. ${ }^{1}$

Unfortunately, technological difficulties in fabricating CPBG structures rapidly increase with decreasing wavelength for which a CPBG is required. Despite the research activities of a large number of experimental groups, achievement of a CPBG below infrared (IR) wavelengths for both two- and three-dimensional (3D) photonic structures is still elusive, mainly because the required dielectric contrast $\delta$ to open a CPBG is rather high. ${ }^{7}$ Here $\delta=\varepsilon_{\max } / \varepsilon_{\min }$, where $\varepsilon_{\text {max }}\left(\varepsilon_{\min }\right)$ is the dielectric constant of a material component, used to fabricate a photonic crystal, with the largest (smallest) value of $\varepsilon$. For the best geometries $\delta \approx 5$ is required. ${ }^{4,8}$ Already this threshold value of $\delta$ excludes the majority of semiconductors and other materials, such as (conducting) polymers, from many useful photonic crystal applications. However, for applications one needs a sufficiently large CPBG to leave a margin for gap-edge distortions due to omnipresent defects. ${ }^{9}$ Let us define $g_{w}$ as the gap width to midgap frequency ratio, $\Delta \omega / \omega_{c}$. Then in order to achieve $g_{w}$ larger than $5 \%, \delta \geq 9.8$ and $\delta \geq 12$ is required for a diamond ${ }^{8}$ and face-centered-cubic (fcc) structure, ${ }^{7}$ respectively. This leaves only a couple of materials for photonic crystal applications at near infrared and optical wavelengths. ${ }^{10}$ 
Surprisingly enough, it will be demonstrated here that there is a way to create a sizeable and robust CPBG in the near infrared and in the visible even for a photonic crystal which contains more than $97 \%$ of a material with the refractive index below $n=1.45$, i.e., that of silica glass or a polymer. The trick is to place small inclusions of a low absorbing metal in the right dielectric structure. It turns out that the effect of small inclusions of a low absorbing metal on the photonic band-gap structure is very strong for a parent diamond dielectric structure. In the latter case, the actual metal volume fraction $f_{m}$ needed to open a CPBG of more than $5 \%$ depends on the available material dielectric constant $\varepsilon$ and can be kept below $1 \%$ for $\varepsilon_{s}=4$ (Fig. 5 below). Given a metal-volume fraction $f_{m}$, the strongest effect on CPBG was found when both spheres in the lattice primitive cell contained identical metal cores. Surprisingly, the inclusions have the biggest effect for the dielectric constant $\varepsilon \in[2,12]$, which is a typical dielectric constant at near infrared and in the visible for many semiconductors and polymers. Given the desired gap width to midgap frequency ratio of $5 \%$, the smallest absorption was found when only one of the two spheres in the lattice primitive cell contained a metal core, i.e., for a zinc-blende structure, even though the required $f_{m}$ was typically twice as large as that for a diamond structure. This result suggests that, given the metal filling fraction $f_{m}$, absorption is reduced due to a reduction of near-field electromagnetic energy transfer between the metal cores with an increased separation of the metal islands in the structure. For a zinc-blende structure, absorption within a CPBG of $5 \%$ can be kept below $2.6 \%$ for $\lambda \geqslant 750 \mathrm{~nm}$. The results on the absorption are by far the best which have been demonstrated for a 3D metallo-dielectric structure with a CPBG. They are an order of magnitude better than for the case of a fcc lattice of metal coated spheres. ${ }^{11}$ Our results on the absorption compare well to the best results for one-dimensional and twodimensional metallo-dielectric photonic crystals which show absorptance of $\approx 1 \%$ and $\approx 3 \%$, respectively, at $\lambda$ $\approx 600 \mathrm{~nm}^{12}$ Photonic band-structure calculations also revealed a surprising scalinglike behavior of our metallodielectric diamond and zinc-blende structures, which is only intrinsic to purely dielectric structures. The scalinglike behavior means that once a CPBG is found, the CPBG can be open for any wavelength, simply by scaling all the sizes of a structure-an extremely useful property from a practical point of view.

A metal-core dielectric-shell sphere morphology seems to play an essential role in the effect of small metal inclusions on photonic band structure. If the same volume of a metal is spread homogeneously within the spheres, no CPBG is found. Similarly, a diamond structure of small metal nanospheres embedded in a dielectric matrix shows a much smaller CPBG and much higher absorptance for a comparable $f_{m}$ than either a diamond or a zinc-blende close-packed structure of metal-core dielectric-shell spheres in air. On purely experimental grounds, only the case of spheres with a metal core is investigated here. Usually, a metal shell around a dielectric core is formed by an aggregation of small metallic nanoparticles. The shell has to be around $20 \mathrm{~nm}$ thick before it becomes complete. ${ }^{13}$ With an emphasis on photonic structures in the near infrared and in the visible, the $20 \mathrm{~nm}$ shell thickness then would mean a rather high threshold value of the metal filling fraction $f_{m}$ (of the order of $5 \%$ ). On the other hand, it is much easier to tune the metal filling fraction $f_{m}$ from zero to a few percent by coating small metal nanoparticles with a dielectric in a controlled way. ${ }^{14}$ Moreover, a dielectric shell is necessary to prevent aggregation of the metallic particles by reducing the Van der Waals forces between them. In the latter case, a coating of roughly $20 \mathrm{~nm}$ is required. Having metal cores also means that an active light-emitting dielectric material (semiconductor or polymer) is not isolated as in the case of metal shells. Hence crystals of metal core-dielectric shell particles can perform more easily some useful optoelectronic functions.

Before proceeding with the photonic band-structure calculation of metallo-dielectric diamond and zinc-blende structure of spheres, it was necessary to recalculate the photonic band structure of the diamond lattice of dielectric spheres. The previous plane-wave method (PWM) calculations of the photonic band structure of diamond ${ }^{4}$ and zinc-blende ${ }^{15}$ lattices of dielectric spheres were found to be plagued by large errors. Therefore, after describing the method of our calculation in Sec. II, we discuss and analyze the differences with respect to earlier photonic band-structure calculations for a diamond lattice of dielectric spheres by Ho, Chan, and Soukoulis. ${ }^{4}$ The effect of small inclusions of a low absorbing metal on the photonic band structure of a diamond and a zinc-blende structure is investigated in Sec. III. In Sec. III A the dependence of CPBG's on the metal volume fraction $f_{m}$ is investigated. The dependence of gaps on either the shell dielectric constant in the diamond case, or on the dielectric constant of the dielectric sphere in the zinc-blende case, is summarized in Sec. III B. A surprising scalinglike behavior of the metallo-dielectric structures is then discussed in Sec. III C. Reflectance, transmittance, and absorptance of light incident on the metallo-dielectric structures is dealt with in Sec. III D. Most of the results will be shown for the case of silver cores. However, as discussed in Sec. IV A, where aluminum, copper, and gold cores are examined, the results so obtained are almost insensitive to the type of metal used, provided that the sphere radius is larger than $\approx 250 \mathrm{~nm}$. If the sphere radius is smaller, most of the results are still qualitatively valid. We then end up with a discussion in Sec. IV and conclusions in Sec. V.

\section{BENCHMARKING DIAMOND STRUCTURE OF DIELECTRIC SPHERES}

Indisputably, one of the hallmarks in the study of photonic band-gap structures is an article by Ho, Chan, and Soukoulis. ${ }^{4}$ It opened a way for the fabrication of the first photonic structure with a complete photonic band gap (CPBG) (Ref. 5) and advanced the field considerably. Its main conclusion is that, regarding a CPBG, a diamond structure of (overlapping) spheres fares much better than a simple fcc structure: (i) a CPBG opens between the second and third bands (the eighth to ninth bands for an inverted fcc structure), and, consequently, is much more stable against disorder, ${ }^{9}$ (ii) the threshold value of the dielectric contrast $\delta$ 


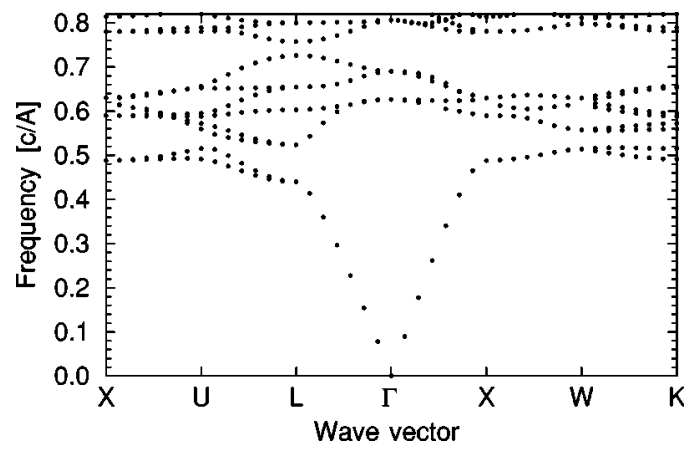

FIG. 1. Photonic band structure for a close-packed diamond lattice of spheres of dielectric constant $\varepsilon=12.96$ in air (the same set of parameters as in Ref. 4). Two CPBG open in the spectrum with the respective gap to midgap frequency ratios of $1.3 \%$ and $4.2 \%$. Frequency is in units [c/A], where $c$ is the speed of light in vacuum and $A$ is the lattice constant of a conventional cubic unit cell of the diamond lattice. If you prefer to use wavelength, the values on the $y$ axis correspond to those of $A / \lambda$, where $\lambda$ is the vacuum wavelength.

to open the CPBG is 4 ( 8.2 for an inverted fcc structure ${ }^{7}$ ), and (iii) the CPBG is significantly larger $(15 \%$ and $5 \%$, for the respective diamond and fcc close-packed lattices of spheres with a dielectric contrast $\delta=12.96$ ).

Results of our calculations are summarized in Figs. 1 and 2. They were obtained using the photonic Korringa-KohnRostocker (KKR) method $^{7,16,17}$ (see also Appendix A). The KKR method can be used for scatterers of arbitrary shape ${ }^{18,19}$ and is optimized for lattices of spheres. The photonic bandgap structure was calculated with the value of the angularmomentum cutoff $l_{\max }=9$. Stability of the Ewald summation was thoroughly checked. Convergence of the method with increasing $l_{\max }$ is demonstrated in Fig. 3. The two lowest bands in Fig. 1 (but not the higher ones) agree well with those calculated in Ref. 15. In agreement with Refs. 4 and 16 no CPBG was found for the case of a diamond lattice of nonoverlapping air spheres in a dielectric. However, for the inverse case of a diamond lattice of nonoverlapping dielectric spheres in air, the earlier results of Ho, Chan, and Soukoulis ${ }^{4}$ on the photonic band structure were found to be nonconverged. Take, for example, the test case of a closepacked diamond lattice of nonoverlapping dielectric spheres

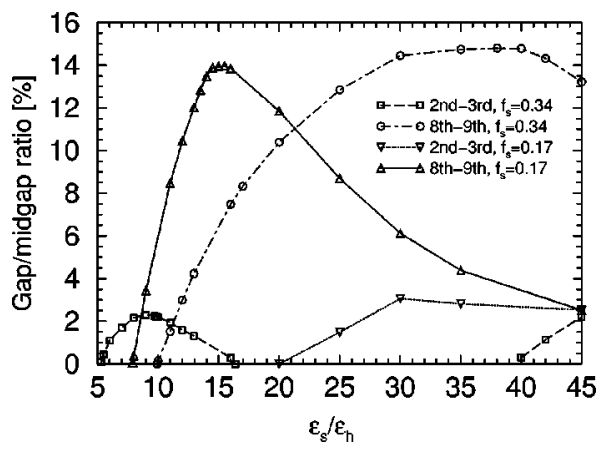

FIG. 2. Gap width to midgap frequency ratio for a diamond lattice of dielectric spheres as a function of the dielectric contrast $\varepsilon_{s} / \varepsilon_{h}$ for sphere-filling fractions $f_{s}=0.17$ and $f_{s}=0.34$.

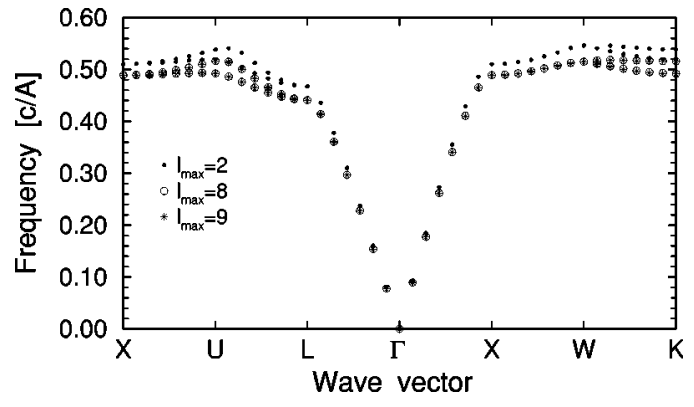

FIG. 3. Convergence of the band structure in Fig. 1 as a function of the cutoff $l_{\text {max }}$.

with the dielectric constant $\varepsilon_{s}=12.96$ in air (Fig. 1). A nonconvergence of the results of Ref. 4 is already witnessed by an unusually large deviation of the effective refractive index $n_{e f f}$, as calculated from the band structure in the L direction, from $n_{e f f}^{M G}=\sqrt{\varepsilon_{e f f}}$, where $\varepsilon_{e f f}$ is calculated by the Garnett
formula,

$$
\varepsilon_{e f f} \approx \varepsilon_{h}(1+2 f \alpha) /(1-f \alpha),
$$

which usually provides a very good fit to $n_{\text {eff }}$. Here $f$ is the sphere volume fraction ( $f=0.34$ for closed-packed diamond and our zinc-blende lattices of spheres) and $\alpha$ is a sphere polarization factor $\left[\alpha=\left(\varepsilon_{s}-\varepsilon_{h}\right) /\left(\varepsilon_{s}+2 \varepsilon_{h}\right)\right.$, where $\varepsilon_{s}$ and $\varepsilon_{h}$ are the dielectric constant of sphere and of host medium, respectively]. In our case, the effective refractive index $n_{\text {eff }}$ $=1.604$ is only $10 \%$ larger than $n_{\text {eff }}^{M G}=1.456$. For comparison, the upper edge of the bands at the $L$ point in Ref. 4 seems to be almost $20 \%$ lower than in our case, resulting in more than a $30 \%$ deviation from $n_{e f f}^{M G}$. Not surprisingly, six years after Ref. 4 the PWM (see Fig. 4 of Ref. 15) yielded for the same test case a second to third CPBG width of only $\approx 8 \%$, half of that presented in Ref. 4. In the meantime, Sözüer, Haus, and Inguva, ${ }^{21}$ p. 13971 , again using the PWM, argued that the second to third CPBG is less than $3.5 \%$, and they called for a recalculation of their results using more efficient methods, such as the photonic KKR method.

For a diamond lattice of nonoverlapping dielectric spheres, i.e., for sphere filling fraction $f_{s}$ varying from 0 to the close-packed case $f_{c p}=0.34$, two CPBG's can occur simultaneously (see Fig. 1), between the second to third bands, and, as in an inverted fcc case, between the eighth and ninth bands. ${ }^{8}$ According to Fig. 2, the photonic band structure of a diamond lattice of nonoverlapping dielectric spheres shows a much more complex behavior of CPBG's than a fcc lattice. For a fcc lattice of air spheres in a dielectric, the calculated gap width to midgap frequency ratio $g_{w}$ of the eighth to ninth CPBG is monotonically increasing with the increasing dielectric contrast $\varepsilon_{h} / \varepsilon_{s}$ and shows a saturated behavior for different filling fractions (see Fig. 4 in Ref. 7). A remarkable feature of a diamond lattice of nonoverlapping dielectric spheres is a kind of gap competition. According to Fig. 2, a CPBG first increases with the dielectric contrast $\varepsilon_{s} / \varepsilon_{h}$, but, at some dielectric contrast shortly before another $\mathrm{CPBG}$ starts to open, the CPBG begins to decrease and it may eventually disappear. As the dielectric contrast $\varepsilon_{s} / \varepsilon_{h}$ increases further, the first CPBG may reappear again, with its reap- 
pearance being signaled by a decrease of the second CPBG. Fig. 2 illustrates that kind of behavior for $f_{s}=0.17$ and $f_{s}$ $=f_{c p}$. Contrary to previous calculations, ${ }^{4}$ the second to third CPBG is found not to be the dominant one. It satisfies the bound of $3.5 \%$ by Sözüer, Haus, and Inguva (see Ref. 21, p. 13971 ) not only for $\varepsilon_{s} \approx 13$ but apparently for all $\varepsilon_{s}$. For $f_{s}=f_{c p}$, the second to third CPBG only persists for $\varepsilon_{s}$ $\in[5.2,16.3]$ and does not exceed 2.3\% (for $\varepsilon_{s}=9$ ). The second to third CPBG is then absent till $\varepsilon_{s}=40$, where it reappears again. We disagree with Ref. 21 that the second to third CPBG vanishes for $f<f_{c p}$. It is true that, for $\varepsilon_{s}=12.96$, the second to third CPBG closes already for $f_{s}=32 \%$, i.e., for $f_{s}$ only $2 \%$ smaller than the close-packed case. However, with increasing $\varepsilon_{s}$ the second to third CPBG can appear at smaller and smaller filling fractions. For instance, for $\varepsilon_{s} \approx 20$ the second to third CPBG begins to open for $f_{s}=17 \%$, but again its $g_{w}$ does not exceed $2.9 \%$, satisfying the $3.5 \%$ bound. ${ }^{21}$ The dominant CPBG is the eighth to ninth CPBG. For $\varepsilon_{s}$ $=12.96$, the eighth to ninth CPBG persists down to $f_{s}$ $=4 \%$. For $f_{s}=17 \%$ and $\varepsilon_{s}=12.96$ it can reach $12 \%$, and it reaches its maximum of $\approx 14 \%$ for $\varepsilon_{s}=15.5$. For $f_{s}=f_{c p}$ it reaches a maximum of $\approx 14.7 \%$ for $\varepsilon_{s}=35$. However, the threshold value of $\varepsilon_{s}$ for its opening is 7.9, comparable to that for an inverted fcc lattice. ${ }^{7}$ Hence, regarding a CPBG, a diamond lattice of nonoverlapping dielectric spheres fares better than a fcc structure of air spheres in a dielectric, ${ }^{7}$ but the advantage is much smaller than has been previously thought.

An argument to persuade the reader about the correctness of our results is that they can be reproduced, with some minor differences, by the PWM based MIT $a b$ initio program. ${ }^{22}$ However, unlike the case of a simple lattice (one scatterer per lattice primitive cell), ${ }^{7}$ for the case of a diamond lattice of dielectric spheres, even when using the PWM based MIT ab initio program, ${ }^{22}$ one has to take a much larger number of plane waves than expected to reach a convergence comparable with the photonic KKR method. As shown by Megens, ${ }^{23}$ to reach convergence of the photonic band structure of a diamond lattice of dielectric spheres within $1 \%$, the number of plane waves $N_{c}$ has to exceed 32768 (cf. Ref. 4) and still an extrapolation $N \rightarrow \infty$ (Ref. 21) has to be performed. Hence, the $N \rightarrow \infty$ extrapolation ${ }^{21}$ might also be necessary to ensure a convergence for the MIT $a b$ initio program ${ }^{22}$ when modeling systems with sharp material discontinuities for other complex lattices. Note that $N_{c}$ is two orders of magnitude larger than $N_{h c s}=339$ which reproduces the results of Ref. 4 for the whole range of filling fractions $0 \leqslant f \leqslant 1 .{ }^{23}$ It turns out that some other results computed in the early days of the plane-wave expansion method can be imprecise and plagued by large errors also, an example being Ref. 24.

\section{EFFECT OF METAL INCLUSIONS ON PHOTONIC BAND STRUCTURE}

In this section, the effect of small inclusions of a low absorbing metal on the photonic band-gap structure for a parent close-packed diamond and zinc-blende dielectric structure of spheres is investigated. The close-packed case of spheres in air was chosen because this is one of the most probable cases to be fabricated either by "do-it-yourself organization" 25 or by a microrobotic technique. ${ }^{26}$ At the same time, even when starting from colloidal suspension, ${ }^{27}$ one expects to use dried structures in air for practical applications which will most probably consist of touching spheres (close-packed structures). A metal core will be placed inside either both dielectric spheres in the lattice primitive cell, or only one single sphere in the lattice primitive cell. In the former case, the resulting structure will be a diamond structure, whereas in the latter case the structure becomes a zincblende structure. In the following we only consider the special case of zinc-blende structures of spheres, when both spheres in the lattice primitive cell have identical radii.

To reach convergence of bands with $1 \%$ precision in the metallo-dielectric case, the photonic KKR method ${ }^{7,17,28}$ with the angular momentum cutoff parameter $l_{\max }=8$ was used. The KKR method is best suited to deal with highly dispersive scatterers. Computational time with and without the dielectric constant dispersion is the same and only depends on the angular momentum cutoff parameter (see also Appendix A). As in earlier work, ${ }^{11,28,29}$ only the real part of the material dielectric constant was used when the photonic band structure was calculated. Reflectance, transmittance, and absorptance were calculated by adapting available computer code, ${ }^{30}$ which is based on the layer photonic KKR (LKKR) method. ${ }^{31}$ In the latter case, both the real and imaginary part of the material dielectric constant were used. The dielectric constant of a metal was chosen according to Ref. 32. Above the vacuum wavelength of $\approx 1900 \mathrm{~nm}$, Palik's data for aluminum, copper, and gold are no longer available. In this case a Drudelike fit to a metal dielectric constant was used with parameters determined by Ordal et al. ${ }^{33}$ (Ordal's parameters have also been used by El-Kady et al. $^{34}$ to fit a metal dielectric constant from far infrared down to optical wavelengths.) The same procedure for the band structure and absorption calculations has also been employed in an earlier work on metallo-dielectric structures. ${ }^{11,28,29}$ It is true that a complex dielectric constant turns Bloch eigenvalues into complex resonances in the lower half of the complex frequency plane. ${ }^{36}$ However, it can be shown that the imaginary part of a low absorbing metal has only little influence on the projections of the complex resonances on the real frequency axis. ${ }^{37}$ This can also be shown by a direct comparison of the "projected" band structure against reflection and transmission calculations (see Sec. III D). Note in passing that a comparison of theory and experiment using the photonic KKR methods has so far been more than satisfactory. ${ }^{29,35}$

Photonic band-structure calculations demonstrated that several CPBG's can open in the spectrum. Depending on the metal filling fraction $f_{m} \leqslant 10 \%$ and a typical dielectric constant $\varepsilon \in(1,20]$ of a dielectric at near infrared and in the visible, a diamond structure can have CPBG's between the second and third and eighth and ninth bands, whereas a zincblende structure can have CPBG's between the second and third, fifth and sixth, and eighth and ninth bands. ${ }^{15}$ Figure 4 shows the photonic band structure for a close-packed zincblende lattice of spheres in air. One of the two spheres in the primitive lattice cell is a silver core $-n_{s}=1.45$ (silica) shell 


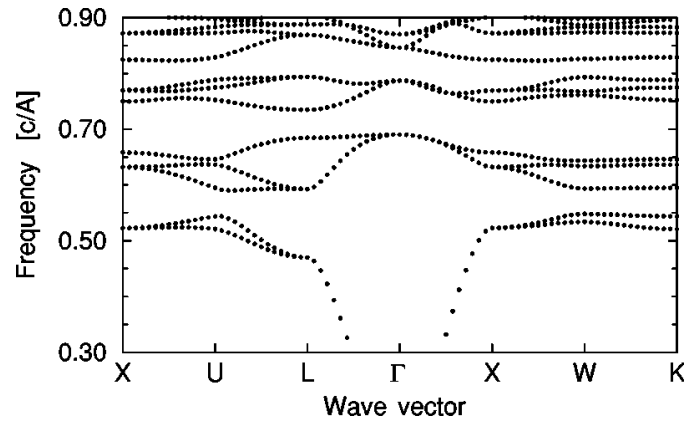

FIG. 4. Photonic band structure for a close-packed zinc-blende lattice of spheres in air. One of the two spheres in the primitive lattice cell has a silver core and dielectric shell with refractive index $n_{s}=1.45$ (silica) with the core to total sphere radii ratio $r_{c} / r_{s}$ $=0.75\left(f_{m}=7.2 \%\right)$, whereas the other is a homogeneous dielectric sphere with $\varepsilon=12$ of the same radius $r_{s}=80 \mathrm{~nm}$. Frequency is in units [c/A], as in Fig. 1.

sphere with $r_{c} / r_{s}=0.75\left(f_{m}=7.2 \%\right)$-whereas the other is a homogeneous dielectric sphere with $\varepsilon=12$ of the same radius $r_{s}=80 \mathrm{~nm}$. The frequency is in units [c/A], as in Fig. 1. By comparing Fig. 1 and Fig. 4 it is obvious that as the result of the metal inclusions much larger second to third and eighth to ninth CPBG's open. There is also an additional CPBG between the fifth and sixth bands. Metal inclusions seem to align band edges at the $L$ and $W$ points, and the degenerate band edge of the third and fourth bands at the $L$ point raises above the band edge of the third band at the $U$ point.

In the following subsection, the dependence of the gaps on the metal volume fraction is investigated. The dependence of gaps on either the shell dielectric constant in the diamond case, or on the dielectric constant of the dielectric sphere in the zinc blende case, is summarized in Sec. III B. A surprising scalinglike behavior of the metallo-dielectric structures is then discussed in Sec. III C. Reflectance, transmittance, and absorptance of light incident on the metallo-dielectric structures is dealt with in Sec. III D.

\section{A. Dependence of gaps on the metal volume fraction}

Photonic band-structure calculations revealed a strong effect of metal inclusions on the CPBG between the second and third bands. Given a metal volume fraction $f_{m}$, the largest second to third CPBG opens for a close-packed diamond lattice of metal core-dielectric shell spheres in air (Fig. 5), followed by a close-packed zinc-blende structure (Figs. 6, 7). Unlike in the purely dielectric case, which has been discussed in Sec. II, in the presence of small inclusions of a low absorbing metal the dominant CPBG for a diamond lattice is that between the second and third bands (Figs. 8, 9). The second to third CPBG is the most important CPBG, because it is the most stable against disorder. ${ }^{9}$ In the zinc-blende case, the second to third CPBG is also much larger than in the purely dielectric case and is a dominant $\mathrm{CPBG}$ for $\varepsilon$ $\in[1,9]$ (Figs. 10, 11). For higher $\varepsilon$ the dominant CPBG becomes the fifth to sixth CPBG (Figs. 10, 11). Photonic band-structure calculations revealed several remarkable fea-

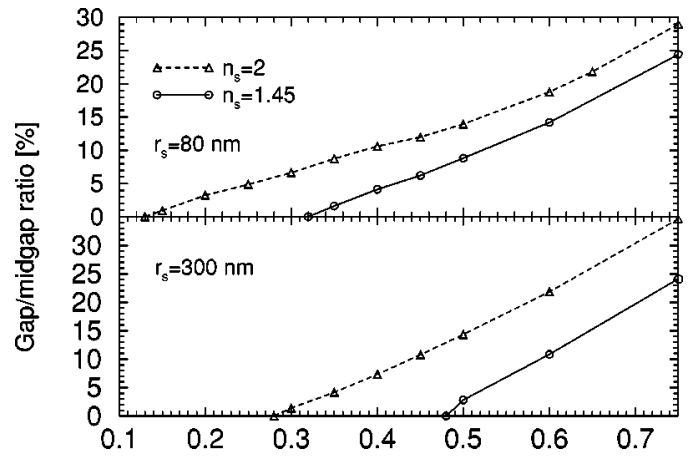

FIG. 5. Calculated gap width to midgap frequency ratio of the second to third CPBG for a close-packed diamond lattice of dielectric $n_{s}=1.45$ (silica) and $n_{s}=2(\mathrm{ZnS})$ coated silver spheres in air. The upper graph is for the sphere radius $r_{s}=80 \mathrm{~nm}$ and the lower graph is for the sphere radius $r_{s}=300 \mathrm{~nm}$. The gap to midgap ratio is plotted as a function of the metal core radial filling fraction $r_{c} / r_{s}$. Metal-volume fraction is then $f_{m}=0.34 \times\left(r_{c} / r_{s}\right)^{3}$.

tures of the diamond and zinc-blende metallo-dielectric structures. First, a strong increase of the second to third CPBG with $f_{m}$, once a threshold value of $f_{m}$ is reached. Figure 5 shows that if the silver cores are placed inside spheres with a refractive index $n_{s}=1.45$ (silica) and a radius of $80 \mathrm{~nm}$, the second to third CPBG below $600 \mathrm{~nm}$ opens for $f_{m} \approx 1.1 \%$ and reaches $5 \%$ already for $f_{m} \approx 2.5 \%$. If the sphere refractive index $n_{s}$ increases further and approaches the threshold refractive index contrast of $\approx 2.3$, for which the second to third CPBG of the parent diamond structure of non-overlapping dielectric spheres begins to open (see Fig. $2)$, the required metal $f_{m}$ 's rapidly decrease. For $n_{s}=2(\mathrm{ZnS})$ spheres with a silver core, the second to third CPBG begins to open already for $r_{c} / r_{s}=0.13\left(f_{m} \approx 0.07 \%\right)$ and reaches $5 \%$ already for $r_{c} / r_{s} \approx 0.26\left(f_{m} \approx 0.6 \%\right)$. Figure 6 shows that in the zinc blende case one needs almost twice as large $f_{m}$ as in the diamond case to obtain a comparable second to third CPBG. For example, to obtain the second to third CPBG of $5 \%$ in the case when silver cores are only placed inside a single $n_{s}=1.45$ (silica) sphere in the lattice primitive

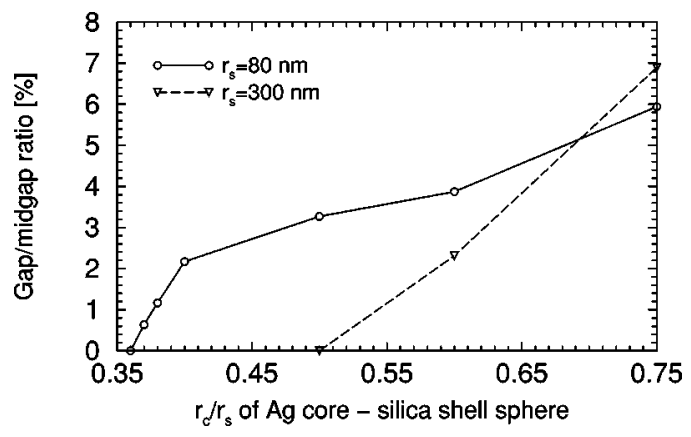

FIG. 6. Calculated gap width to midgap frequency ratio of the second to third CPBG for a close-packed metallo-dielectric zincblende lattice of spheres in air. Lattice primitive cell contains a silica sphere and a silver core $-n_{s}=1.45$ (silica) shell sphere of the same radii. $g_{w}$ is plotted as a function of the ratio $r_{c} / r_{s}$ of the silver core $-n_{s}=1.45$ (silica) shell sphere for the cases $r_{s}=80 \mathrm{~nm}$ and $r_{s}=300 \mathrm{~nm}$. Metal-volume fraction is then $f_{m}=0.17 \times\left(r_{c} / r_{s}\right)^{3}$. 


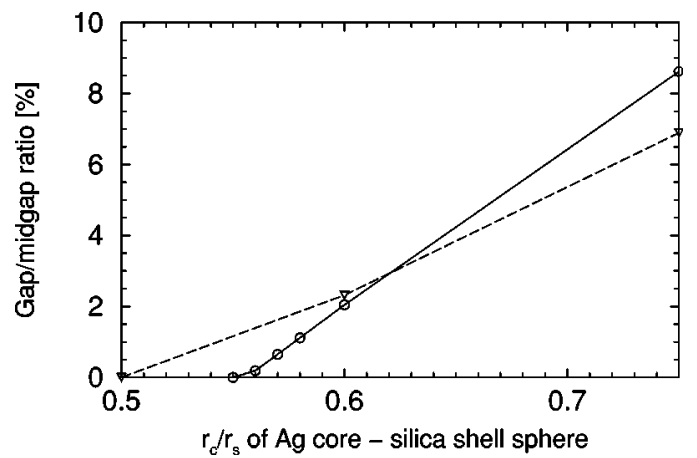

FIG. 7. Calculated gap width to midgap frequency ratio of the second to third CPBG for a zinc-blende lattice of spheres in air for the sphere radius of $r_{s}=300 \mathrm{~nm}$. One of the two spheres in the lattice primitive cell is a silver core $-n_{s}=1.45$ (silica) shell sphere, whereas the other is a dielectric sphere of the same radius. Figure shows the effect of the second sphere on the second to third CPBG which is either a $n_{c}=2(\mathrm{ZnS})$ core $-n_{s}=1.45$ (silica) shell dielectric sphere with fixed $r_{c} / r_{s}=0.60$ (dashed line) —or a homogeneous $n=1.45$ (silica) sphere (solid line).

cell, one needs $f_{m} \approx 5 \%$. Figure 7 shows the effect of the dielectric sphere morphology on the second to third CPBG, which is either a $n_{c}=2(\mathrm{ZnS})$ core-silica shell dielectric sphere with fixed $r_{c} / r_{s}=0.60$ (dashed line) —or a homogeneous silica sphere (solid line). The other sphere in the lattice primitive cell is a silver core $-n_{s}=1.45$ (silica) shell sphere.

\section{B. Dependence of gaps on the dielectric constant}

Figures $8,9,10$, and 11 illustrate the effect of a varying dielectric constant on a CPBG. In the diamond case, the shell dielectric constant is varied. In the zinc-blende case, one of the spheres in the lattice primitive cell remains a silver core- $n_{s}=1.45$ (silica) shell sphere with fixed $r_{c} / r_{s}=0.75$ $\left(f_{m}=7.2 \%\right)$, and we vary the dielectric constant of the dielectric sphere. Quite amazingly, small metal inclusions have the biggest effect for $\varepsilon \in[2,12]$ (cf. Figs. 8, 10, and 11),

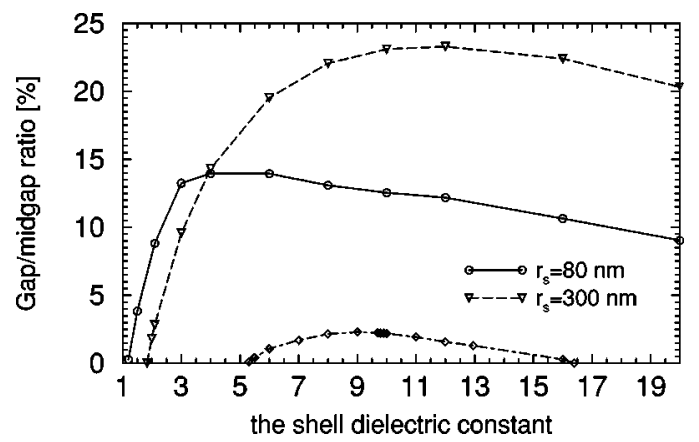

FIG. 8. Gap width to midgap frequency ratio of the second to third CPBG for a close-packed metallo-dielectric diamond lattice of silver core-dielectric shell spheres with $r_{c} / r_{s}=0.5\left(f_{m}=4.25 \%\right)$ in air as a function of the shell dielectric constant for the cases $r_{s}$ $=80 \mathrm{~nm}$ and $r_{s}=300 \mathrm{~nm}$. For a comparison, the dot-dashed line with diamonds shows the gap to midgap frequency ratio of the second to third CPBG of a close-packed diamond lattice of purely dielectric spheres.

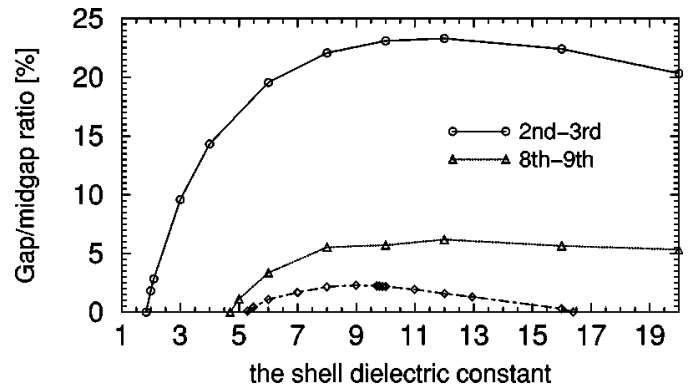

FIG. 9. Gap width to midgap frequency ratio of the second to third and eighth to ninth CPBG's for a close-packed metallodielectric diamond lattice of silver core-dielectric shell spheres with $r_{c} / r_{s}=0.5\left(f_{m}=4.25 \%\right)$ in air as a function of the shell dielectric constant for $r_{s}=300 \mathrm{~nm}$. For a comparison, the dot-dashed line with diamonds shows the gap to midgap frequency ratio of the second to third CPBG of a close-packed diamond lattice of purely dielectric spheres.

which is a typical dielectric constant in the near infrared and in the visible for many materials such as semiconductors and polymers. The dependence of the CPBG's on the dielectric constant is very complex. Figures 9, 10, and 11 show that the second to third CPBG remains the largest CPBG for $\varepsilon \leqslant 9$. According to Figs. 10 and 11, for zinc blende structures and $\varepsilon \geqslant 9$, the second to third CPBG becomes smaller than the fifth to sixth CPBG. The eighth to ninth CPBG remains small for both diamond and zinc-blende structures. As already seen for a diamond structure of nonoverlapping dielectric spheres (see Fig. 2), ${ }^{8}$ if $\varepsilon$ increases beyond a certain threshold value, the second to third CPBG begins to narrow and may even disappear. Such a narrowing of the second to third CPBG with an increasing dielectric constant seems a characteristic feature of the diamond and zinc-blende structures. Figure 11 shows the same kind of "gap competition" which has been

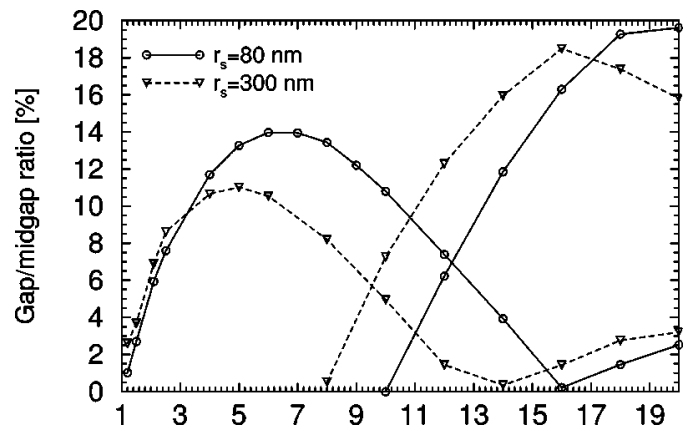

FIG. 10. Calculated gap width to midgap frequency ratio of the second to third and the fifth to sixth CPBG's for a close-packed metallo-dielectric zinc-blende lattice of spheres in air. One of the two spheres in the primitive lattice cell is a silver core $-n_{s}=1.45$ (silica) shell sphere with $r_{c} / r_{s}=0.75$-whereas the other is a homogeneous dielectric sphere of the same radius $r_{s}\left(f_{m}=7.2 \%\right)$. The gap/midgap ratio is plotted as a function of the dielectric constant of the homogeneous sphere for the cases $r_{s}=80 \mathrm{~nm}$ and $r_{s}$ $=300 \mathrm{~nm}$. With increasing dielectric constant of the homogeneous sphere, first the second to third CPBG opens. The appearance of the fifth to sixth CPBG roughly coincides with the point after which the second to third CPBG begins to narrow. 


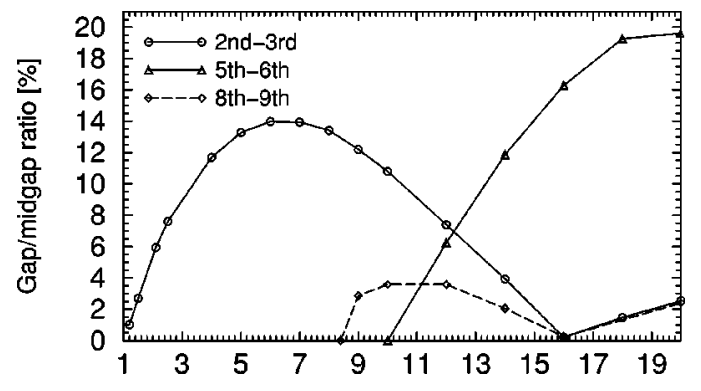

FIG. 11. Calculated gap width to midgap frequency ratio of the second to third, fifth to sixth, and eighth to ninth CPBG's for a close-packed metallo-dielectric zinc-blende lattice of spheres in air. One of the two spheres in the primitive lattice cell is a silver core$n_{s}=1.45$ (silica) shell sphere with $r_{c} / r_{s}=0.75$-whereas the other is a homogeneous dielectric sphere of the same radius $r_{s}=80 \mathrm{~nm}$ $\left(f_{m}=7.2 \%\right)$. The gap/midgap ratio is plotted as a function of the dielectric constant of the homogeneous sphere. Note that around $\varepsilon$ $\approx 16$ only the fifth to sixth CPBG remains open.

first observed in the purely dielectric case (see Fig. 2): a CPBG first increases with increasing dielectric constant, but at some dielectric contrast shortly before another CPBG starts to open, the CPBG begins to decrease and it may eventually disappear.

\section{Scalinglike behavior}

A further remarkable feature of the metallo-dielectric structure is a surprising scalinglike behavior, which is intrinsic only to ideal dispersionless structures. Given the metal filling fraction $f_{m}$, the best scalinglike behavior is observed in our zinc-blende structures (see Figs. 12, 13, 14, 17). A scalinglike behavior is expected once the metal $\varepsilon$ at a given midgap wavelength becomes sufficiently large and negative. Indeed, on the single-scatterer level it makes rather little difference if the real part of the metal $\varepsilon=-200$ or $\varepsilon=-\infty$, the limit of a perfect metal. Figure 15 shows that differences in

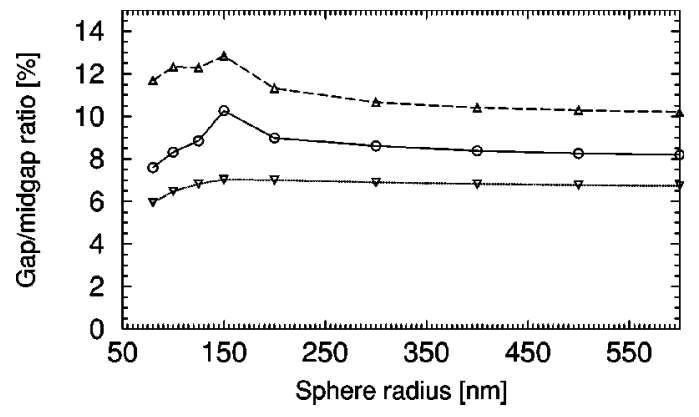

FIG. 12. Calculated gap width to midgap frequency ratio of the second to third CPBG for a close-packed metallo-dielectric zincblende lattice of spheres in air as a function of the sphere radius. One of the two spheres in the primitive lattice cell is a silver core$n_{s}=1.45$ (silica) shell sphere with $r_{c} / r_{s}=0.75$-whereas the other is a dielectric sphere of the same radius $r_{s}\left(f_{m}=7.2 \%\right)$. The dashed line corresponds to the case when the second sphere is a homogeneous $n=2(\mathrm{ZnS})$ sphere, the solid line is the case of a $n_{c}=2(\mathrm{ZnS})$ core $-n_{s}=1.45$ (silica) shell sphere with fixed $r_{c} / r_{s}=0.60$, and the dotted line is for the case of a homogeneous $n=1.45$ (silica) sphere.

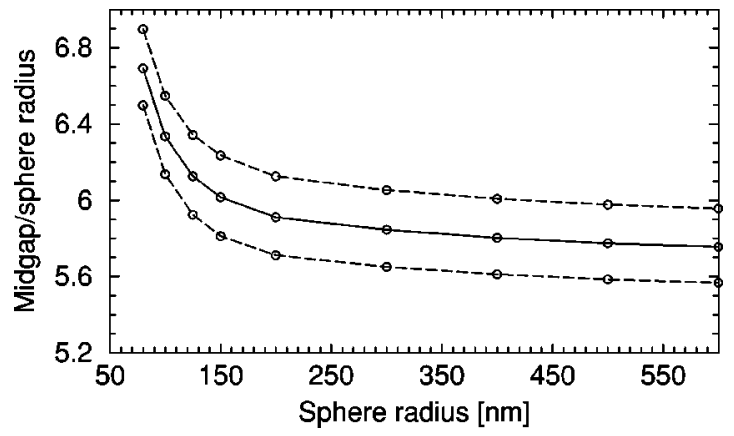

FIG. 13. An example of scaling of the midgap wavelength (solid line) of the second to third CPBG for a close-packed zinc-blende lattice of spheres in air with the sphere radius. Gap edges are plotted by dashed lines. One of the two spheres in the lattice primitive cell is a silver core $-n_{s}=1.45$ (silica) shell sphere with fixed $r_{c} / r_{s}=0.75$-and the other sphere is a homogeneous $n=1.45$ (silica) sphere of the same radius $\left(f_{m}=7.2 \%\right)$. Gap width to midgap frequency ratio of the second to third CPBG of the structure is shown by dotted line in Fig. 12.

the sphere extinction efficiency rapidly decrease with an increasing sphere radius. There is only a little difference in the extinction efficiencies of $r_{s}=300 \mathrm{~nm}$ and $r_{s}=600 \mathrm{~nm}$ spheres. One can, therefore, view the preceding figures involving $r_{s}=300$ spheres as a limiting case of a perfect metal. For silver inclusions, the midgap position and the second to third CPBG edges follow almost perfect scaling down to $r_{s}$ $=200 \mathrm{~nm}$. For $r_{s}<200 \mathrm{~nm}$, the gap width and gap edges begin to depend stronger and stronger on decreasing $r_{s}$. However, if the midgap wavelength is plotted in $\mathrm{nm}$ against sphere radius, one would see a linear dependence down to $r_{s}=80 \mathrm{~nm}$ (see Figs. 14, 16), for which the second to third CPBG midgap wavelength is typically below $600 \mathrm{~nm}$ and the real part of silver $\varepsilon \approx-10$. Therefore, in Fig. 13 the ratios of the midgap and gap edges of the second to third

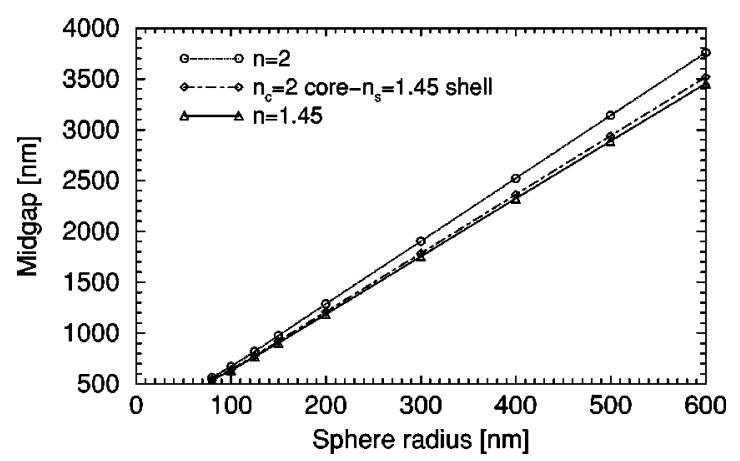

FIG. 14. An example of scaling of the midgap wavelength of the second to third CPBG for a close-packed zinc-blende lattice of spheres in air with the sphere radius. One of the two spheres in the lattice primitive cell is $n_{s}=1.45$ (silica) shell sphere with a silver core with fixed $r_{c} / r_{s}=0.75$, whereas the other is a purely dielectric sphere of the same radius $\left(f_{m}=7.2 \%\right)$. The latter is either a homogeneous $n=1.45$ (silica) sphere, a homogeneous $n=2$ (ZnS) sphere, or a $n_{c}=2(\mathrm{ZnS})$ core $-n_{s}=1.45$ (silica) shell sphere with fixed $r_{c} / r_{s}=0.6$. Gap width to midgap frequency ratio of the second to third CPBG of these structures is shown in Fig. 12. 


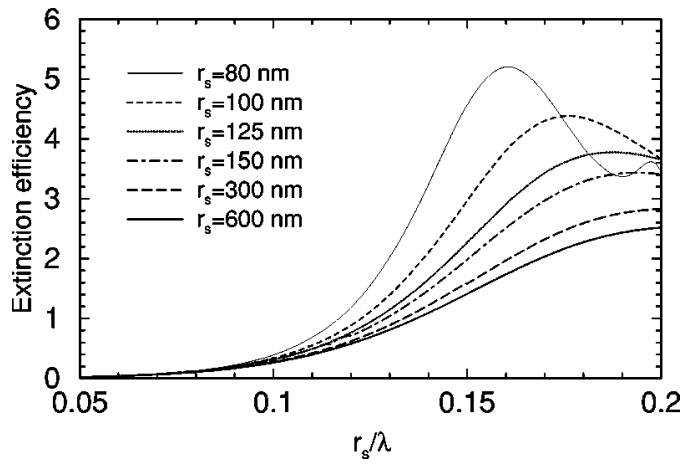

FIG. 15. The extinction efficiency for a silver core $-n_{s}=1.45$ (silica) shell sphere with fixed $r_{c} / r_{s}=0.75$ for different sphere radii.

CPBG to the sphere radius are plotted to make deviations from the scalinglike behavior visible at all. This scalinglike property is very useful from a practical point of view. It means that once a CPBG is found, with some midgap wavelength $\lambda_{c}$, the CPBG can be centered at any other wavelength by a simple scaling of all the sizes of a structure.

\section{Reflectance, transmittance, absorptance}

In order to further investigate optical properties of our photonic structures, reflectance, transmittance, and absorptance were calculated. By reflectance, $\mathcal{R}$, and transmittance, $\mathcal{T}$, we mean total reflected and transmitted field (the specular and all propagating higher diffraction orders), respectively. Absorptance, $\mathcal{A}$, is then defined as the total loss in the structure, $\mathcal{A}=1-\mathcal{R}-\mathcal{T}$. Reflectance, transmittance, and absorptance were calculated by adapting available computer code, ${ }^{30}$ which is based on the photonic LKKR method. ${ }^{31}$ The same method was also used in Refs. 11 and 29. To reach convergence within $1 \%$ around the second to third $\mathrm{CPBG}, l_{\max }$ $=6$ was used.

For a metallo-dielectric structure it is characteristic that, beyond a certain crystal thickness, reflectance, transmittance, and absorptance within a CPBG change only negligibly with an increasing crystal thickness. ${ }^{38}$ As a function of the crystal thickness, $l$, absorptance behaves as

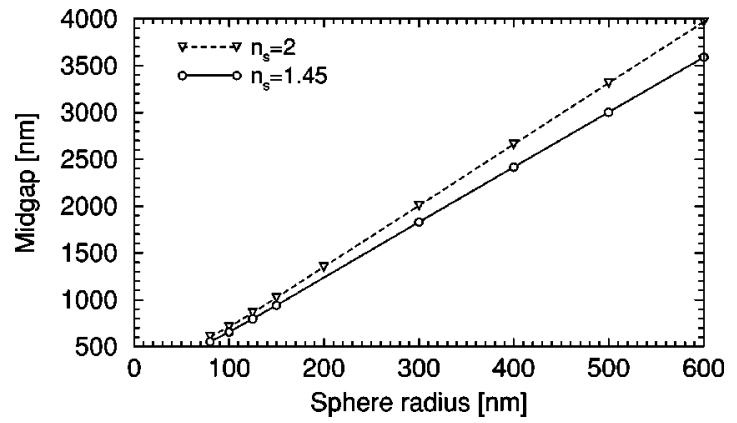

FIG. 16. An example of scaling of the midgap wavelength of the second to third CPBG for a close-packed diamond lattice of coated silver spheres in air with the sphere radius. Spheres are either $n_{s}$ $=1.45$ (silica) coated silver spheres with fixed $r_{c} / r_{s}=0.6$, or $n_{s}$ $=2(\mathrm{ZnS})$ coated silver spheres with fixed $r_{c} / r_{s}=0.4$.

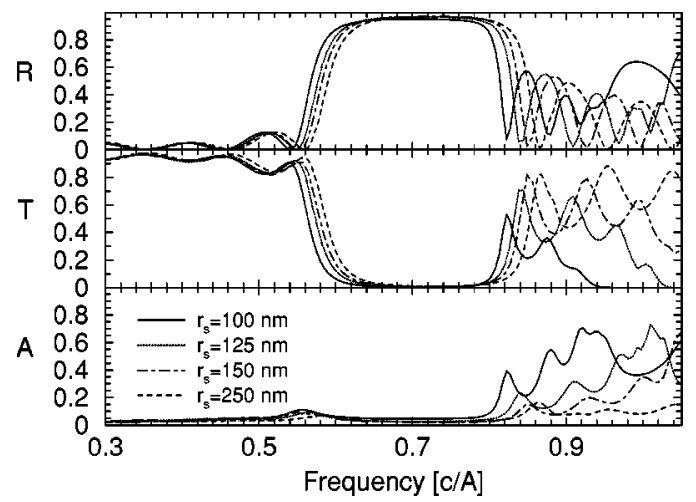

FIG. 17. Reflectance, transmittance, and absorptance of light incident normally on a two unit-cell (12 planes) thick zinc-blende lattice of spheres in air stacked in the (111) direction. The spheres are as in Figs. 12 and 13. Dimensionless frequency is used on the $x$ axis, where $A$ is the lattice constant of a conventional unit cell of the cubic lattice. In all cases, the second to third CPBG lies between $\approx 0.7$ and 0.8 . Gap width to midgap frequency ratio of the second to third CPBG of these structures is shown in Fig. 12, the midgap wavelength of the second to third CPBG is shown in Figs. 13 and 14.

$$
\mathcal{A}(l)=\mathcal{A}_{\text {sat }}\left[1-\exp \left(-l / l_{A}\right)\right],
$$

where $l_{A}$ is an "absorption" length and $\mathcal{A}_{\text {sat }}$ is the saturated absorptance. For some CPBG's, $\mathcal{A}_{\text {sat }}$ can only be a few percent. This saturated behavior can easily be explained as follows. Because of a CPBG, light can only penetrate the first few layers of a crystal. Hence, an addition of further layers beyond the penetration length has only minor influence. As a direct consequence of the saturation, absorptance per crystal thickness decreases approximately linearly with the crystal thickness, $\mathcal{A}(l) / l \approx \mathcal{A}_{s a t} / l$. For a high metal-volume fraction, as for a fcc photonic crystal, such a saturated behavior can already be established after three crystal planes. ${ }^{11,29}$ In our case, the metal-volume fraction is almost 20 times smaller. Consequently, the saturation (with two digit precision) is established after 12 crystal planes for a normal incidence in the (111) crystal direction, or, after two unit-cell thickness. The (111) crystal direction has been chosen because this choice makes it possible to compare the properties of our photonic crystals against those of fcc photonic crystals, which properties have been studied in the same crystal direction. ${ }^{11,29}$ It is useful to remind that the (111) direction corresponds to the $\Gamma-L$ direction in the notation of special points on the surface of the Brillouin zone. Hence, for our diamond and zincblende structures, reflection and transmission measurements in the (111) direction probe the slice of band structure (see Figs. 1 and 4) which consists of the line parallel to the $y$ axis which intersects the $x$ axis at the $L$ point.

In Fig. 17 reflectance, transmittance, and absorptance of light incident normally on a two-unit-cell (12 planes) thick zinc-blende lattice of spheres in air stacked in the (111) direction is shown. Note first a scalinglike behavior of reflectance, transmittance, and absorptance in Fig. 17. The spheres are as in Figs. 12, 13, and 14. The AABBCC stacking sequence is depicted in Fig. 18. The gap width to midgap frequency ratio of the second to third CPBG of these configu- 


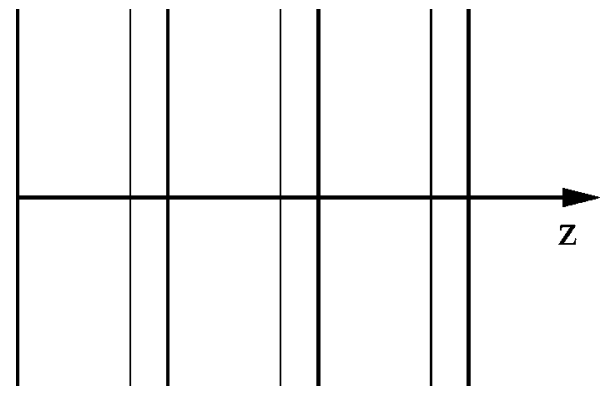

FIG. 18. AABBCC stacking of our diamond and zinc-blende structures in the (111) direction. Stacking is along the body diagonal of the conventional unit cell of the cubic lattice. Lines perpendicular to the stacking $z$ direction indicate hexagonal planes of spheres with the primitive lattice vectors $\mathbf{a}_{1}$ and $\mathbf{a}_{2}$. Nearest-neighbor distance of hexagonal planes depicted by the same line is $1 / 3$ of the body diagonal. Spheres in the planes separated by $1 / 4$ of the body diagonal have identical $x y$ positions. Spheres in the planes separated by $1 / 12$ of the body diagonal are shifted by $\left(\mathbf{a}_{1}+\mathbf{a}_{2}\right) / 3$ with respect to each other. The close-packed radius is $1 / 8$ of the body diagonal.

rations has been shown in Fig. 12. The midgap wavelength of the second to third CPBG has been shown in Figs. 13 and 14.

Calculations based on the LKKR method ${ }^{30,31}$ showed that the saturated absorptance within a CPBG can be kept at very small levels. Given the desired gap width of $5 \%$, the smallest absorption was found for close-packed zinc-blende structures, even though the required $f_{m}$ was typically twice as large as that of the close-packed diamond structure. According to Fig. 17, the saturated absorptance within a CPBG of $\geqslant 5 \%$ can be kept below $5 \%$ for a CPBG centered at $\lambda$ $\approx 600 \mathrm{~nm}$ and is less than $2.6 \%$ for a CPBG centered at $\lambda$ $\geqslant 750 \mathrm{~nm}$. This should be tolerable in most practical applications. The results on the absorption are by far the best which have been demonstrated for a 3D metallo-dielectric structure with a CPBG. They are an order of magnitude better than for the case of a fcc lattice of metal-coated spheres. ${ }^{11}$ (Although we have used the same method as in Refs. 11,29 to calculate absorption, when recalculating the results in Ref. 11 for a fcc lattice of metal-coated dielectric spheres, a much larger absorptance was obtained.) Our results for the saturated absorptance compare well to the best results for $1 \mathrm{D}$ and 2D metallo-dielectric photonic crystals which show a saturated absorptance of $\approx 1 \%$ and $\approx 3 \%$ respectively, within a CPBG centered at $\lambda \approx 600 \mathrm{~nm}^{12}$

Note in passing that within the second to third CPBG and for normal incidence in the (111) direction, almost $99 \%$ reflection goes into the specular beam. As the angle of incidence increases, the specular reflectivity decreases and gradually more light is reflected in nonspecular directions.

\section{DISCUSSION}

\section{A. Use of others metals as a sphere core}

So far, when presenting our results on the effect of small inclusions of a low absorbing metal on the photonic band structure of diamond and zinc-blende photonic crystals, we

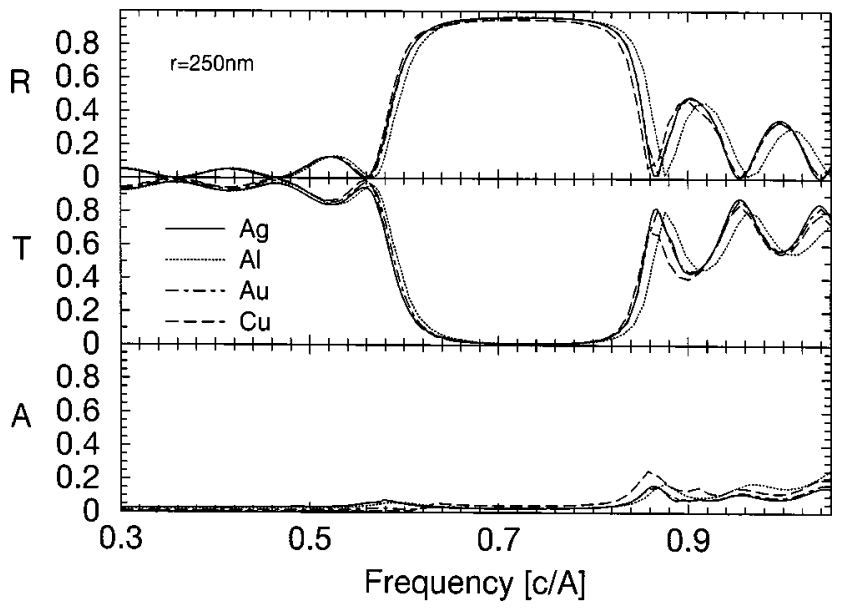

FIG. 19. Reflectance, transmittance, and absorptance of light incident normally on a two-unit-cell (12 planes) thick zinc-blende lattice of spheres in air stacked in the (111) direction as a function of frequency for geometrically identical cores made of different metals. The geometry of $r=250 \mathrm{~nm}$ spheres is the same as in Fig. 17. Dimensionless frequency is used on the $x$ axis, where $A$ is the lattice constant of a conventional unit cell of the cubic lattice. In all cases, the second to third CPBG lies between $\approx 0.7$ and 0.8 .

have used exclusively silver. This noble metal guarantees the best properties of metallo-dielectric photonic crystals in the near infrared and in the visible. ${ }^{11,28,29}$ However, almost identical results have also been obtained for aluminum, copper, and gold cores, provided that sphere radius $r_{s} \geqslant 250 \mathrm{~nm}$ (see Fig. 19). For $r_{s}<250 \mathrm{~nm}$ spheres, the results for different metal can be increasingly different with decreasing $r_{s}$, nevertheless qualitative features remain the same (see Fig. 20).

This behavior is explained as follows. When the scalinglike behavior was discussed in Sec. III C, it was argued that one can view the results involving $r_{s} \geqslant 250 \mathrm{~nm}$ spheres as those corresponding to a limiting case of a perfect metal, when metal dielectric constant is $\varepsilon=-\infty$. Indeed, Fig. 15 showed that differences in the single-sphere extinction efficiency rapidly decrease with an increasing sphere radius. Therefore, one expects only minor differences for diamond

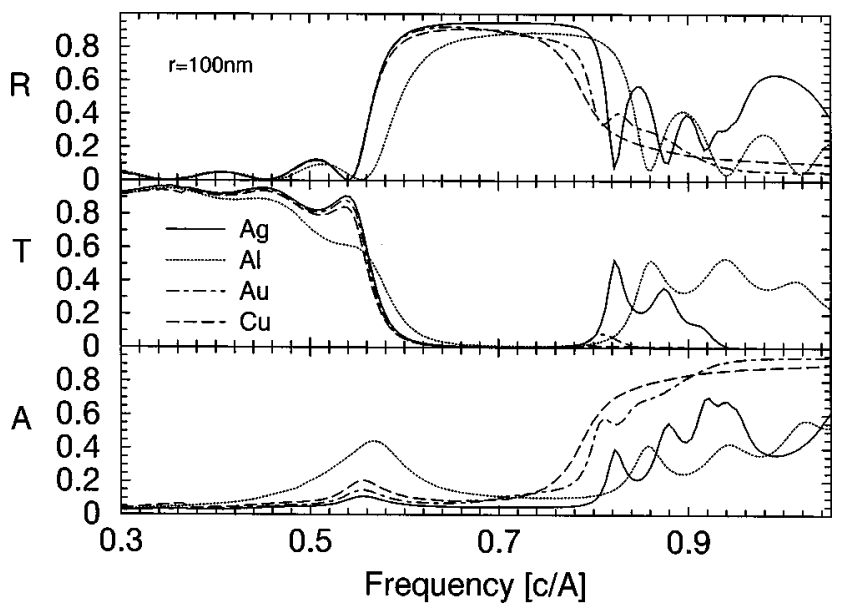

FIG. 20. The same as in Fig. 19 except that the sphere radius is now $100 \mathrm{~nm}$. 
and zinc-blende photonic crystals with $r_{s} \geqslant 250 \mathrm{~nm}$ spheres when gold, copper, or some other metal is used instead of silver, with the other lattice and sphere parameters being identical. These expectations are fully confirmed by exact calculations, and the results are displayed in Figs. 19 and 20. A similar behavior, i.e., only a slight change in absorption when gold or copper has been used in place of silver, has also been found for a fcc structure. ${ }^{34}$

\section{B. Fec vs diamond and zinc-blende structures}

Obviously, small metal inclusions do not open a CPBG in every dielectric structure. For example, a simple fcc lattice of dielectric spheres with a metal core requires $f_{m} \approx 50 \%$ to open a CPBG. ${ }^{28,29}$ For a fcc lattice of metal-coated dielectric spheres, the required metal filling fraction $f_{m}$ is slightly lower but still very high $\left(\approx 40 \%{ }^{11}\right)$ and still almost 40 times larger than in our case. Therefore, not surprisingly, when going further to shorter and shorter wavelengths, one is facing an increasing absorption: at $\lambda \approx 600 \mathrm{~nm}$ the absorption exceeds $30 \%$ even within a CPBG. Although such a metallodielectric fcc structure could provide a CPBG (Refs. 11, 28 and 29) at near infrared, the extension to the visible is difficult. A probable reason for such a big effect of small metallic inclusions on the photonic band gaps of the diamond and zinc-blende structures compared to a fcc structure is because the former have much better photonic band-gap properties already in the limiting cases of purely dielectric and purely metallic spheres. In the limit of dielectric spheres in air, 4,15 the threshold value of the dielectric contrast to open the second to third CPBG for a diamond structure is only $5.2{ }^{8}$ whereas a fcc structure does not have any $\mathrm{CPBG}$, irrespective of the sphere dielectric constant. ${ }^{7,21}$ In the opposite limit of pure metallic spheres, the second to third CPBG for the diamond lattice is huge. For silver, depending on the sphere radius $r_{s}$, it can stretch from $60 \%\left(r_{s}=80 \mathrm{~nm}\right)$ to $75 \%$ $\left(r_{s} \geqslant 300 \mathrm{~nm}\right) .^{8,29}$ This is consistent with a previous estimate of $g_{w} \geqslant 60 \%$ for the case of an ideal metal $\left(\varepsilon_{s}=-\infty\right) .{ }^{39}$ On the other hand, for a fcc lattice a higher CPBG opens (between the fifth and sixth bands) and is smaller $(\approx 40 \%){ }^{29}$

\section{Behavior of the second to third CPBG with an increasing metal volume fraction}

In order to explain the opening of a CPBG with an increasing metal volume fraction, the following argument has been used by Zhang et al.: ${ }^{29}$ Low-frequency waves propagate through a 3D metallo-dielectric structure as in an effective medium characterized by the effective dielectric constant $\varepsilon_{e f f} \cdot{ }^{20}$ This low-frequency pass band will have zero group velocity when the wave vector approaches the Brillouin-zone boundary where the wave vector $k \approx \pi / a, a$ being the lattice constant. Thus the highest frequency $\omega_{l}$ of this low frequency pass band $\approx c /\left(a \sqrt{\varepsilon_{e f f}}\right)$. On the other hand, metal cores inscribe a void with a scale of the same order as $a$, and the fundamental resonance modes in the void should have frequencies of the order $\omega_{u} \approx c / a$. These voids are always connected together by channels, so that the modes can hop from one void to another forming a pass band. Since $\varepsilon_{e f f}$ increases when the metal-volume fraction increases, it may be possible to have $\omega_{l}<\omega_{u}$ when the metal-volume fraction exceeds a certain threshold value. Then, assuming that there are no other bands between the low-frequency pass and hopping bands, a CPBG should be formed between $\omega_{l}$ and $\omega_{u}$. However, Zhang et al. ${ }^{29}$ applied their argument to a fcc lattice where a CPBG opens between the fifth and sixth bands, i.e., the band on the lower edge of the CPBG is not a low-frequency pass band. It seems more natural to reconsider their argument for our case of diamond and zinc-blende photonic crystals of coated spheres.

The Garnett formula ${ }^{20}$ (1) yields $\varepsilon_{\text {eff }}$ for a cubic lattice of both homogeneous and coated spheres. One has only to substitute a corresponding polarization factor for $\alpha$. In the case of a coated sphere with a single coating, embedded in the host medium characterized by the dielectric constant $\varepsilon_{h}$, let us denote the dielectric constant and radius of the sphere core (shell) by $\varepsilon_{c}$ and $r_{c}\left(\varepsilon_{s}\right.$ and $\left.r_{s}\right)$, respectively. Defining $x$ $=r_{c}^{3} / r_{s}^{3}, \quad \alpha_{c}=\left(\varepsilon_{c}-\varepsilon_{s}\right)\left(\varepsilon_{c}+2 \varepsilon_{s}\right), \quad$ and $\alpha_{s}=\left(\varepsilon_{s}-\varepsilon_{h}\right)\left(\varepsilon_{s}\right.$ $\left.+2 \varepsilon_{h}\right)$, the polarization factor $\alpha$ of a coated sphere with a single coating can be written as ${ }^{7}$

$$
\alpha=\frac{\alpha_{s}+x \alpha_{c}\left(\varepsilon_{h}+2 \varepsilon_{s}\right) /\left(\varepsilon_{s}+2 \varepsilon_{h}\right)}{1+2 x \alpha_{c} \alpha_{s}} .
$$

Here $0 \leqslant x \leqslant 1$ and $0 \leqslant \alpha_{j}<1$. Note that $\alpha_{c}\left(\alpha_{s}\right)$ coincides with the polarization factor of a homogeneous sphere with the dielectric constant $\varepsilon_{c}\left(\varepsilon_{s}\right)$ embedded in the host medium characterized by the dielectric constant $\varepsilon_{s}\left(\varepsilon_{h}\right){ }^{20}$ (Polarization factors of a homogeneous sphere are also recovered from $\alpha$ in one of the following limits: $x \rightarrow 0, x \rightarrow 1$, and $\varepsilon_{c}$ $\rightarrow \varepsilon_{s} \cdot{ }^{7}$ )

For a diamond lattice of close-packed metal-core dielectric-shell spheres in air, $\varepsilon_{\text {eff }}$, as calculated from Eqs. (1) and (3), increases from $\approx 1.3$ up to $\approx 1.9$, i.e., by only a factor of $\approx 1.5$, when $r_{c} / r_{s}$ increases from 0.1 to 0.75 (irrespective of the real part of the dielectric constant of the metal core when varied between -50 and -500 , since the metalvolume fraction is kept rather small). Therefore, in our case, the change in $\varepsilon_{e f f}$ with an increasing metal-volume fraction $f_{m}$ is by no means dramatic. Yet the behavior of the second to third CPBG edges, as shown in Fig. 21, seems to be qualitatively in agreement with the argument by Zhang et al. ${ }^{29}$ For $r_{s}=80 \mathrm{~nm}$ spheres, both CPBG edges first decrease with increasing $r_{c} / r_{s}$. However, when $r_{c} / r_{s}$ increases beyond $\approx 0.5$, the upper second to third CPBG edge slows down its decrease and becomes approximately constant, whereas the lower edge of the second to third CPBG continues in its monotonic decrease. The case of $r_{s}=300 \mathrm{~nm}$ spheres shows a qualitatively different behavior of the upper second to third CPBG edge, which frequency increases with increasing $r_{c} / r_{s}$. This behavior is exactly what one would expect according to the argument by Zhang et al. ${ }^{29}$ In the case of $r_{s}=300 \mathrm{~nm}$ spheres, the second to third CPBG is located at the frequency range for which silver skin depth $(=c /[2 \omega \operatorname{Im} n(\omega)])$ is only slightly above $10 \mathrm{~nm}$, whereas the silver core has a radius between 150-180 nm. Therefore, light can only penetrate a small fraction of the silver core. Consequently, since the void volume between spheres 


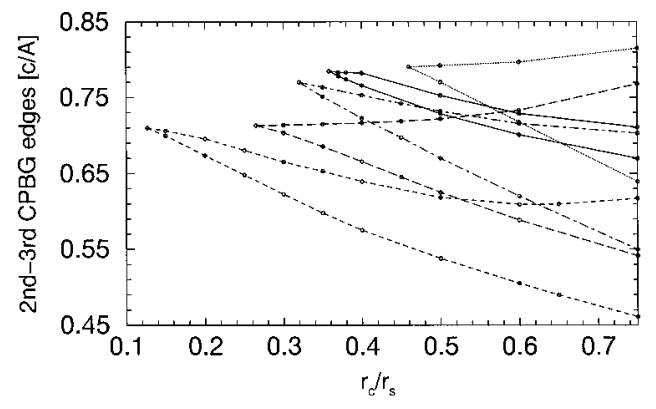

FIG. 21. Behavior of the second to third CPBG edges with an increasing relative radius of the silver core, $r_{c} / r_{s}$. Shown are the cases of a zinc-blende lattice having $\mathrm{Ag} @ \mathrm{SiO}_{2}$ and $\mathrm{SiO}_{2}$ spheres of the same radius $r_{s}=80 \mathrm{~nm}$ in the primitive cell (solid lines), a diamond lattice of silver-core $-n_{s}=1.45$ shell spheres with radii $r_{s}=80 \mathrm{~nm}$ (dot-dashed lines) and $r_{s}=300 \mathrm{~nm}$ (dotted lines), and a diamond lattice of silver-core $-n_{s}=2$ shell spheres with radii $r_{s}$ $=80 \mathrm{~nm}$ (dashed lines) and $r_{s}=300 \mathrm{~nm}$ (long-dashed lines).

shrinks as $r_{c} / r_{s}$ increases, the fundamental resonance modes in the void shift to higher frequencies. In the case of $r_{s}$ $=80 \mathrm{~nm}$ spheres, the second to third CPBG is located at the frequency range for which silver skin depth can be as large as $50 \mathrm{~nm}$, i.e., light can penetrate the entire silver core. Therefore, the "void" part of the argument by Zhang et al. is much less applicable in the $r_{s}=80 \mathrm{~nm}$ case.

\section{Coated vs homogeneous metal spheres}

We have also examined if a large and robust CPBG can open by using homogeneous metal nanospheres arranged on a diamond lattice in a dielectric, with the possibility of fabricating a diamond structure by layer-by-layer deposition. According to Fig. 18, the sphere projections on the stacking $z$ direction in neighboring planes do not overlap if the sphere radius $r_{s} \leqslant r_{c p} / 3$, where $r_{c p}$ is the sphere radius in the closepacked case. Therefore, if $r_{s} \leqslant r_{c p} / 3$ it would be possible to fabricate a diamond lattice by depositing first a hexagonal plane of spheres on a substrate and then filling in the interstitial by a dielectric until all spheres are embedded in a dielectric layer. One would then repeat the procedure layer by layer and grow gradually a diamond lattice. Unfortunately, the constraint $r_{s} \leqslant r_{c p} / 3$ means that the resulting sphere filling fraction $f_{s} \leqslant 1.26 \%$, which is too small to achieve a large and robust CPBG with homogeneous metal nanospheres in a dielectric. Indeed, no CPBG was found for a diamond structure of homogeneous metal nanospheres with $r_{s} \leqslant 0.45 r_{c p}\left(f_{m} \approx 3.1 \%\right)$ embedded in a dielectric host with the dielectric constant $\varepsilon=2.1,3,4$, and 6.25. Photonic band-structure calculations revealed that one needs $f_{s}=f_{m}$ $\approx 10 \%$ to open the second to third CPBG of $5 \%$ with metal nanospheres in silica. For comparison, note that for a closepacked diamond structure of $n_{s}=1.45$ dielectric spheres with a metal core and radius of $80 \mathrm{~nm}$, the second to third CPBG opens for $r_{c}=0.32 r_{c p}$, and for $r_{c}=0.45 r_{c p}$ the second to third CPBG reaches almost $5 \%$ (Fig. 5). This suggests that a metal core-dielectric shell sphere morphology plays a crucial role in the formation of CPBG's. Indeed, if the same volume of a metal is spread homogeneously within the spheres, no

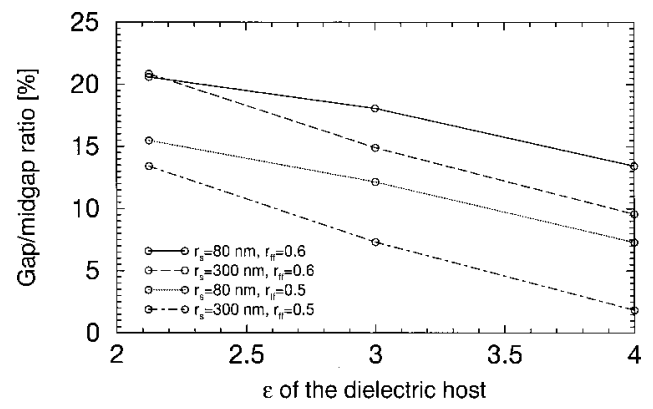

FIG. 22. Calculated gap width to midgap frequency ratio of the second to third CPBG for a close-packed metallo-dielectric diamond lattice of spheres as a function of the host dielectric constant. The dependence is shown for the sphere radii $r_{s}=80$ and $300 \mathrm{~nm}$. Spheres have a silver core and dielectric shell with $\varepsilon=8$. The core to total sphere radii ratio, $r_{f f}$, is either $r_{c} / r_{s}=0.5\left(f_{m}=4.25 \%\right)$, or $r_{c} / r_{s}=0.6\left(f_{m}=7.34 \%\right)$. For $r_{c} / r_{s}=0.4\left(f_{m}=2.2 \%\right)$ and the dielectric shell with $\varepsilon=4$, the second to third CPBG is closed in this range of the host dielectric constant.

CPBG opens in the spectrum. In the latter case, the sphere dielectric constant was calculated using the Garnett formula (1). ${ }^{20}$

A dielectric contrast between the dielectric shell and the host dielectric medium also seems to be an important factor. Figure 22 shows the dependence of the second to third CPBG for a close-packed metallo-dielectric diamond lattice of spheres of radii $r_{s}=80 \mathrm{~nm}$ and $300 \mathrm{~nm}$ on the host dielectric constant. Spheres have a silver core and dielectric shell with $\varepsilon=8$, with the core to total sphere radii ratio either $r_{c} / r_{s}=0.5\left(f_{m}=4.25 \%\right)$, or $r_{c} / r_{s}=0.6\left(f_{m}=7.3 \%\right)$. The shell dielectric constant has to be sufficiently large to open a CPBG. For example, for the dielectric shell with $\varepsilon=4$ and $r_{c} / r_{s}=0.4\left(f_{m}=2.2 \%\right)$, the second to third CPBG is closed in the range of the host dielectric constant in Fig. 22.

\section{E. Absorption}

Similarly, as in the case of a photonic crystal of small $r_{s}$ $=5 \mathrm{~nm}$ Drude plasma spheres, ${ }^{40}$ one observes (see Figs. 17, 19, and 20) that the absorptance peaks coincide with the transmittance peaks. An explanation for this behavior can already be traced down to the case of a homogeneous slab of metal. In the latter case, one can show that neither the imaginary part of the metal dielectric constant nor the inverse skin depth (absorption coefficient) characterize properly the absorption of an incident light. Indeed, the absorption coefficient is a measure of absorption of only that light which has entered the metal. Higher transmittance means higher probability of light being absorbed. Hence, a proper measure of the degree of absorption of an incident light is rather a product of the transmittance and the absorption coefficient.

In the purely dielectric case ${ }^{30}$ and in the case of a fcc crystal of small $r_{s}=5 \mathrm{~nm}$ Drude plasma spheres with sufficiently large relaxation time of conduction electrons and a low volume fraction $(f=0.1),{ }^{40}$ transmittance peaks occur when the effective half wavelength, $\lambda / 2=\pi / k_{\perp}, k_{\perp}$ being the wave vector in the stacking direction, times an integer equals approximately the thickness of the photonic crystal 
slab. Hence, when transmittance is plotted against $k_{\perp}$, one observes a series of almost equidistantly spaced peaks. ${ }^{{ }^{30,40}} \mathrm{It}$ is well known that $d k_{\perp} / d \omega$ can attain very high values near band edges. As a rule of thumb, band edges are located either at the surface, or, in the center $(\mathbf{k}=0$ or $\Gamma$ point $)$ of the Brillouin zone. At the points on the surface of the Brillouin zone with reflection symmetry, frequency eigenvalues satisfy $\omega(\mathbf{k})=\omega(-\mathbf{k})$. For those points $d k_{\perp} / d \omega$ even diverges to infinity. (The latter is equivalent to saying that at such a band edge the group velocity of light approaches zero.) Therefore, if transmittance is plotted against frequency, one observes a considerable increase in the density of transmittance peaks in the immediate vicinity of a frequency gap. However, when the relaxation time of conduction electrons of Drude plasma spheres gradually decreases (what is equivalent to an increasing imaginary part of the dielectric constant), the densely spaced sharp transmittance and absorptance peaks near band edges merge and generate a rather smooth profile. ${ }^{40}$ This is what is also observed in our case, as exemplified in Figs. 17, 19 , and 20, which show no densely spaced and sharp transmission peaks in the proximity of the lower second to third CPBG edge.

A dramatic change in the absorptance at the lower and upper band gap edges is a reminiscent of the Borrmann effect, previously observed in x-ray scattering. ${ }^{41}$ The origin of this effect is that fields in the proximity of band-gap edges approach a standing wave. In the proximity of one band-gap edge, fields are mostly localized in the regions of a low dielectric constant, whereas in the proximity of the other band gap edge fields are mostly localized in the regions of a high dielectric constant. This sort of behavior can already be observed for a simple 1D periodic Bragg stack of dielectric layers. ${ }^{42}$ Figures 17, 19, and 20 suggest that in the proximity of the lower band-gap edge of our zinc-blende structures the electric field is mostly localized in air, whereas in the proximity of the upper band-gap edge the electric field is mostly localized at sphere positions, leading to a significantly higher absorption.

Our results on absorption compare well to the best results for $1 \mathrm{D}$ and $2 \mathrm{D}$ structures. ${ }^{12}$ In these respective cases, the absorption of an optimized metallo-dielectric structure can be $\approx 1 \%$ and $\approx 3 \%$ within a CPBG centered at $\lambda$ $\approx 600 \mathrm{~nm}$. Given the metal filling fraction $f_{m}$, it has been found that absorption is reduced when metal forms isolated islands. ${ }^{34}$ This is an expected result: when metal forms an interconnected network, DC conductivity is nonzero and long-range conduction currents are induced which lead to higher losses. Our results suggest that absorption is reduced further when separation of metal islands increases. Indeed, a larger separation of metal cores in our zinc-blende structure compared to a diamond structure resulted in a significantly reduced absorption. This is probably because of a reduction of near-field electromagnetic energy transfer between the metal cores with an increased separation of the metal islands in the structure.

\section{F. Fabrication}

Whereas a simple fcc structure occurs naturally in colloidal crystals formed by monodisperse colloidal suspensions of

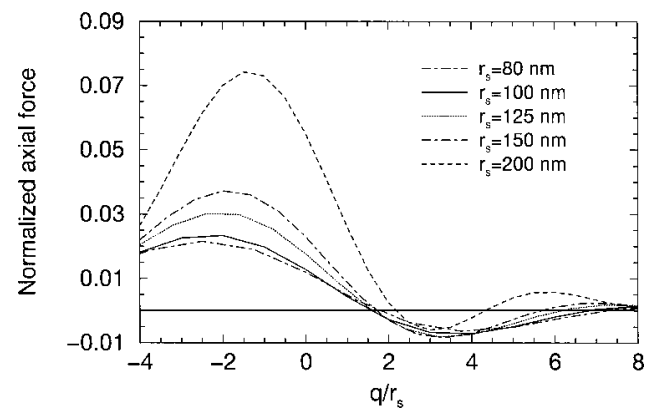

FIG. 23. Normalized axial force plotted against $q / r_{s}$, the center offset from the focus in units of the sphere radius. Silver core$n_{s}=1.45$ (silica) shell spheres with fixed $r_{c} / r_{s}=0.3$ are immersed in water $n_{h}=1.33$. Laser wavelength $\lambda$ increases by $100 \mathrm{~nm}$ from $\lambda=600 \mathrm{~nm}$ for $r_{s}=80 \mathrm{~nm}$ to $\lambda=1000 \mathrm{~nm}$ for $r_{s}=200 \mathrm{~nm}$. In all cases a stable equilibrium is achieved at around $q / r_{s} \approx 2$.

microspheres or via template-directed colloidal crystallization, ${ }^{27}$ diamond and zinc-blende structures are very difficult to fabricate. Nevertheless, several new ways have recently been proposed to make these and other complex structures, ${ }^{25,26,43}$ and therefore diamond and zinc-blende structures deserve our full attention. One of the possibilities is a do-it-yourself organization which, using artificial templates, enables one to create structures beyond thermodynamic limits. ${ }^{25}$ Patterning of a surface can be performed using optical tweezers ${ }^{44}$ and particle manipulation can also be employed in a microrobotic technique. ${ }^{26}$ Difficulties in optical trapping of a metal-core dielectric-shell sphere increase with an increasing core size. There is always a danger that a metal core can be melted in the laser focus. For spheres with a $7.5 \mathrm{~nm}$ gold core and a silica shell with a final radius of $79 \mathrm{~nm}$, optical trapping has been recently demonstrated experimentally in Ref. 44. In Fig. 23 we show that metal-core silica-shell spheres with much larger cores can also be optically trapped. We plot there the axial trapping efficiency $Q$ for silver core $-n_{s}=1.45$ (silica) shell spheres with fixed $r_{c} / r_{s}=0.3$ immersed in water $\left(n_{h}=1.33\right)$. The axial trapping efficiency $Q$, or the dimensionless normalized axial force, when multiplied by the focused laser beam power $P$, determines the resulting axial force $F$ on a sphere immersed in a homogeneous medium with refractive index $n_{h}$,

$$
F=\left(n_{h} / c\right) P Q .
$$

The laser wavelength $\lambda$ is increased by $100 \mathrm{~nm}$ with increasing sphere radius, from $\lambda=600 \mathrm{~nm}$ for $r_{s}=80 \mathrm{~nm}$ to $\lambda=1000 \mathrm{~nm}$ for $r_{s}=200 \mathrm{~nm} . Q$ is plotted against $q / r_{s}$, the center offset from the focus in units of the sphere radius. The beam opening angle of the focused beam was taken to be $\theta_{0}=78$ and the ratio of the objective focal length to the beam waist was set to 0.99 . The normalized axial force $Q$ was calculated by adapting results of Ref. 45. A stable equilibrium location on the beam axis corresponds to the point where the normalized axial force $Q$, considered as a function of $q / r_{s}$, crosses zero with a negative derivative. Fig. 23 shows that the spheres can be optically trapped for all radii, with a stable equilibrium at around $q / r_{s} \approx 2$. Note that the equilibrium point is almost five times further from the focus 
in the current case than in the case of purely dielectric spheres (cf. Fig. 2 of Ref. 45). A larger distance from the focus is convenient experimentally as it reduces the chance of melting a metal core by an intense light.

\section{G. Related work}

In our study we have only considered metal-core dielectric-shell particles which can easily be fabricated. ${ }^{14}$ In this respect, our results regarding a CPBG are not optimized. Probably the required metal-volume fraction can be rendered even smaller than in our case. One way is to use perturbation theory along the lines of Ref. 46. Here it has been demonstrated that a photonic band gap can be enlarged by placing metal insertions at particular positions of a lattice. ${ }^{46}$ However, this work dealt with an enlargement of already existing band gaps, whereas, in our case, small inclusions of a low absorbing metal are used to open a CPBG. Also Ref. 46 only deals with photonic crystals in two dimensions, whereas our work covers 3D photonic crystals. Photonic band gaps of $A B(3)$ and $B-3$ structures of metallo-dielectric spheres have been investigated in Ref. 47. It has been shown that an $A B(3)$ photonic crystal contains a large photonic band gap and a $B-3$ photonic crystal has more than one gap when the filling ratio of metal spheres exceeds a threshold. A complementary case of 3D diamond structures of metal-coated dielectric spheres has recently been discussed in Ref. 48. Such metal nanoshells, consisting of a dielectric core with a metallic shell of nanometer thickness (cca $50 \mathrm{~nm}$ ), can be used to vary the optical resonances of such nanoparticles over hundreds of nanometers in wavelength by varying the relative dimensions of the core and shell. ${ }^{49}$ It has been shown that, under certain conditions, it is possible to center a resonance band, which originates from the single-sphere Mie resonances of metal-coated dielectric spheres, at the middle of the second to third CPBG. The position and width of the resonance-induced in-gap modes can be controlled by making appropriate choices of the dielectric constant of the inner dielectric sphere and thickness of the metal-coating layer, respectively. Such properties can be very useful in making optical band filters as well as microcavity lasers if they are sustained in the presence of dissipation. In the case of metalcore dielectric-shell spheres the tunability of the singlesphere Mie resonances is much smaller. Nevertheless, in the latter case the resonance-induced in-gap modes can be observed for certain diamond and zinc-blende configurations. A study of these resonance-induced in-gap modes in the case of metal-core dielectric-shell spheres will be presented elsewhere. Note that in order to center a single-scatterer resonance band within a CPBG, the presence of a metal is crucial. The latter is impossible in a purely dielectric case for a reasonable dielectric contrast. ${ }^{50}$

\section{CONCLUSIONS}

Diamond and zinc-blende photonic crystals have been studied both in the purely dielectric case and in the presence of small inclusions of a low absorbing metal. In the former case we have shown that earlier plane-wave method calcula- tions of the photonic band structure were not converged. Unlike the case of a simple lattice (one scatterer per lattice primitive cell), ${ }^{7}$ for the case of a diamond lattice of dielectric spheres, even when using the plane-wave method based MIT $a b$ initio program, ${ }^{22}$ one has to take a much higher number of plane waves than expected to reach a convergence comparable with the photonic KKR method. To reach convergence of the photonic band structure of a diamond lattice of dielectric spheres within $1 \%$, the number of plane waves $N_{c}$ has to exceed 32768 (cf. Ref. 4) and still an extrapolation $N \rightarrow \infty$ (Ref. 21) has to be performed. ${ }^{23}$

It has been shown that small inclusions of a low absorbing metal can have a dramatic effect on the photonic band structure of diamond and zinc-blende structures. Several complete photonic band gaps (CPBG's) can open in the spectrum, between the second and third, fifth and sixth, and eighth and ninth bands. The respective metal-volume fractions to open a CPBG and to have a CPBG of 5\% can be more than 40 times and 25 times smaller than in the case of a fcc lattice..$^{11,28,29}$ Unlike in the purely dielectric case, in the presence of small inclusions of a low absorbing metal and for a moderate dielectric constant $(\varepsilon \leqslant 10)$, the largest CPBG turns out to be the second to third CPBG. The second to third CPBG is the most important $\mathrm{CPBG}$, because it is the most stable against disorder. ${ }^{9}$ One can create a sizeable $(\geqslant 5 \%)$ and robust second to third CPBG at the near-infrared and visible wavelengths even for a photonic crystal which contains more than $97 \%$ of low dielectric constant materials $(\varepsilon \leqslant 2.1)$. For diamond and zinc-blende structures of nonoverlapping dielectric and metallo-dielectric spheres, a CPBG begins to decrease with an increasing dielectric contrast roughly at the point where another CPBG starts to open-a kind of gap competition. A CPBG can even shrink to zero when the dielectric contrast increases further (Figs. 2, 10, 11). As a consequence of the gap competition, the inclusions of a low absorbing metal have the biggest effect for the dielectric constant $\varepsilon \in[2,12]$, which is a typical dielectric constant at near infrared and in the visible for many materials, including semiconductors and polymers. Absorptance in the second to third CPBG of $5 \%$ and located above $\lambda \geqslant 750 \mathrm{~nm}$ remains very small $(\leqslant 2.6 \%)$ and is an order of magnitude smaller than in the case of a fcc lattice. ${ }^{11,29}$ Our zinc-blende structures require an almost twice as large $f_{m}$ as diamond structures do to achieve a comparable $\mathrm{CPBG}$, yet they display smaller absorption due to an increased separation of the metal cores. The metallo-dielectric structures display a scalinglike behavior, which makes it possible to scale the CPBG from microwaves down to the ultraviolet wavelengths. Given the metal filling fraction $f_{m}$, this scalinglike behavior is strongest for zinc-blende structures.

Our results imply that any dielectric material can be used to fabricate a photonic crystal with a sizeable and robust CPBG in three dimensions, as long as metal inclusions can be added. These findings (i) open the door for any semiconductor and polymer material to be used as a genuine building block for the creation of photonic crystals with a CPBG and (ii) significantly increase the possibilities for experimentalists to realize a sizeable and robust CPBG at near infrared and in the visible. By relaxing the constraints on the dielec- 
tric constant, the ultimate goal of matching the photonic and electronic band gaps, which is a prerequisite for many applications, ${ }^{2}$ can be achieved.

\section{ACKNOWLEDGMENTS}

I would like to thank my colleagues A. van Blaaderen, A. Imhof, M. Megens, A. Tip, and K. P. Velikov for careful reading of the manuscript and useful comments. SARA computer facilities are also gratefully acknowledged.

\section{APPENDIX A: THE KKR METHOD}

Let $\Lambda$ be a simple (Bravais) periodic lattice. According to the Bloch theorem, propagating wave $\psi$ in a periodic structure with the symmetry $\Lambda$ is characterized by the Bloch momentum $\mathbf{k}$. The latter describes translational properties of $\psi$ by any lattice vector $\mathbf{r}_{s} \in \Lambda$,

$$
\psi\left(\mathbf{r}+\mathbf{r}_{s}\right)=\psi(\mathbf{r}) \exp \left(i \mathbf{k} \cdot \mathbf{r}_{s}\right) .
$$

The Bloch property holds irrespective of the spin of a wave, i.e., is the same for scalar and vector waves. We shall confine ourselves to the case when, outside scatterers, wavefunction $\psi$ satisfies the scalar Helmholtz equation,

$$
\left[\Delta+\sigma^{2}\right] \psi=0,
$$

with $\sigma$ being a positive constant. Let $G_{0 \Lambda}(\sigma, \mathbf{k}, \mathbf{R})$ denote the free-space periodic Green's function of the Helmholtz equation. The latter is defined as

$$
\begin{aligned}
G_{0 \Lambda}(\sigma, \mathbf{k}, \mathbf{R}) & =\sum_{\mathbf{r}_{s} \in \Lambda} G_{0}\left(\sigma, \mathbf{R}-\mathbf{r}_{s}\right) e^{i \mathbf{k} \cdot \mathbf{r}_{s}} \\
& =\sum_{\mathbf{r}_{s} \in \Lambda} G_{0}\left(\sigma, \mathbf{R}+\mathbf{r}_{s}\right) e^{-i \mathbf{k} \cdot \mathbf{r}_{s}},
\end{aligned}
$$

where $\mathbf{R}=\mathbf{r}-\mathbf{r}^{\prime}$ and $G_{0}$ denotes a free-space scattering Green's function of the scalar Helmholtz equation at the points $\mathbf{r}$ and $\mathbf{r}^{\prime}$. In $3 \mathrm{D}$,

$$
G_{0}(\sigma, \mathbf{R})=G_{0}\left(\sigma, \mathbf{r}, \mathbf{r}^{\prime}\right)=-\frac{\exp (i \sigma R)}{4 \pi R},
$$

where $R=|\mathbf{R}|$. Within the Korringa-Kohn-Rostocker (KKR) method, ${ }^{51}$ band structure is determined by solving the KKR secular equation

$$
\operatorname{det}[1-t(\sigma) g(\sigma, \mathbf{k})]=0,
$$

where $t$ is a single-scatterer $T$ matrix and $g$ is the matrix of structure constants. ${ }^{51}$ Both $t$ and $g$ in Eq. (A5) are considered as matrices with matrix elements labeled by pairs of angular momentum numbers $\left(l m, l^{\prime} m^{\prime}\right)$, where $-l \leqslant m \leqslant l$. In the scalar case, for instance, in the case of multiple-scattering scattering of electrons, the matrix elements of $g$ in the angular-momentum basis are defined as expansion coefficients of

$$
\begin{aligned}
G_{0 \Lambda}(\sigma, \mathbf{k}, \mathbf{R})-G_{0}(\sigma, \mathbf{R})= & \sum_{l m, l^{\prime} m^{\prime}} g_{l m, l^{\prime} m^{\prime}}(\sigma, \mathbf{k}) j_{l}(\sigma r) \\
& \times Y_{l m}(\mathbf{r}) j_{l^{\prime}}\left(\sigma r^{\prime}\right) Y_{l^{\prime} m^{\prime}}^{*}\left(\mathbf{r}^{\prime}\right),
\end{aligned}
$$

where $j_{l}$ are the regular spherical Bessel functions, ${ }^{52}$ and $Y_{l m}$ are the conventional spherical harmonics. ${ }^{52}$

It is interesting to note that, to a large extent, the scalar case also covers the scattering of vector and tensorial waves, i.e., waves with a nonzero spin, provided each field component independently obeys the scalar Helmholtz equation (A2) ${ }^{17,31}$ In the vector case of multiple-scattering of electromagnetic waves, $\sigma=\omega \sqrt{\varepsilon_{h}} / c$, where $\omega$ is the angular frequency, $c$ is the speed of light in vacuum, and $\varepsilon_{h}$ is the dielectric constant of the host dielectric medium. Let $L$ $=(\mathrm{lm})$ stand for a multiindex of angular momentum numbers and let $A$ label independent polarizations. The corresponding vector structure constants $G_{A L, A^{\prime} L^{\prime}}$ are obtained from the scalar structure constants $g_{L, L^{\prime}}$ as

$$
\begin{aligned}
G_{A L, A^{\prime} L^{\prime}}= & \sum_{p, p^{\prime}=-1}^{1} \sum_{\alpha, \alpha^{\prime}=-1}^{1} U_{A}(l, m, p, \alpha) \\
& \times g_{l+p, m+\alpha ; l^{\prime}+p^{\prime}, m^{\prime}+\alpha^{\prime}} U_{A^{\prime}}\left(l^{\prime}, m^{\prime}, p^{\prime}, \alpha^{\prime}\right),
\end{aligned}
$$

where the $U_{A}$ 's are group-theoretical coefficients, in the current case determined by the vector-coupling Clebsh-Gordon coefficients. ${ }^{17,31}$ Similarly for the acoustic and elastic waves. In the case of the layer KKR method, ${ }^{17,30}$ which can be viewed as an extension of dynamical x-ray diffraction theory ${ }^{41}$ to infinitely many diffracted beams, one is mainly interested in the reflection, transmission, and absorption properties of finite-width crystals. These properties are determined by the crystal scattering matrix. Roughly speaking, the denominator of the scattering matrix is the matrix which stands behind determinant sign in the KKR secular equation (A5), whereas the nominator of the scattering matrix is rather trivial. Therefore, in a numerical implementation, it is only required to make the following two modifications in the scalar KKR and LKKR numerical codes: (i) to include a single routine which performs the transformation of the scalar structure constants into vectorial structure constants ${ }^{7,17,28}$ and (ii) to use the routine which calculates $t$, the single-scatterer $T$ matrix, appropriate to a given boundary value problem. The same is also true if one wants to adapt the scalar 3D KKR numerical codes which deal with clusters of more than 1000 particles of arbitrary shape and clusters of more than 1000 arbitrary impurities in a crystal. ${ }^{19,53}$ There seems to be no obstacle to have the photonic KKR methods ${ }^{7,17,28,31}$ dealing with clusters of more than 300 particles. The KKR methods can be used for scatterers of arbitrary shape ${ }^{19}$ and are optimized for lattices of spheres.

If $\Lambda$ is not a simple (Bravais) periodic lattice, i.e., there is more than one scatterer in the primitive lattice cell, the matrices $t$ and $g$ in Eq. (A5) become matrices with entries labeled by multiindices $A L \alpha$, where $A$ and $L$ are as before and $\alpha$ runs over all the scatterers in the primitive lattice cell. ${ }^{54}$ 
*Present address: ESTEC/ESA, Electromagnetics Division, P. O. Box 299, NL-2200 AG Noordwijk, The Netherlands. Electronic address: www.amolf.nl/research/photonic_materials_theory/ moroz/moroz.html

${ }^{1}$ V.P. Bykov, Sov. Phys. JETP 35, 269 (1972); Sov. J. Quantum Electron. 4, 861 (1975).

${ }^{2}$ E. Yablonovitch, Phys. Rev. Lett. 58, 2059 (1987).

${ }^{3}$ Proceedings of the NATO ASI School, Photonic Crystals and Localization in the 21st Century, edited by C. M. Soukoulis (Kluwer, Dordrecht, 2001).

${ }^{4}$ K.M. Ho, C.T. Chan, and C.M. Soukoulis, Phys. Rev. Lett. 65, 3152 (1990).

${ }^{5}$ E. Yablonovitch, T.J. Gmitter, and K.M. Leung, Phys. Rev. Lett. 67, 2295 (1991).

${ }^{6}$ A. Mekis, J.C. Chen, I. Kurland, S. Fan, P.R. Villeneuve, and J.D. Joannopoulos, Phys. Rev. Lett. 77, 3787 (1996).

${ }^{7}$ A. Moroz and C. Sommers, J. Phys.: Condens. Matter 11, 997 (1999); A. Moroz, J. Opt. A: Pure Appl. Opt. 1, 471 (1999).

${ }^{8}$ A. Moroz, Photonic Crystals at Near-infrared and Optical Wavelengths, in Organic Optoelectronic Materials, Processing and Devices, Proceedings of the MRS Fall Meeting 2001 (Materials Research Society, Pittsburgh, 2001), Vol. 708.

${ }^{9}$ Z.-Y. Li and Z.-Q. Zhang, Phys. Rev. B 62, 1516 (2000).

${ }^{10}$ B.G. Levi, Phys. Today 52(1), 17 (1999).

${ }^{11}$ Z. Wang, C.T. Chan, W. Zhang, N. Ming, and P. Sheng, Phys. Rev. B 64, 113108 (2001).

${ }^{12}$ A. Moroz, Towards Complete Photonic Band Gap Structures Below Infrared Wavelengths, in Ref. 3, pp. 373-382.

${ }^{13}$ C. Graf and A. van Blaaderen, Langmuir 18, 524 (2002).

${ }^{14}$ L.M. Liz-Marzán, M. Giersig, and P. Mulvaney, Langmuir 12, 4329 (1996); V.V. Hardikar and E.J. Matijević, J. Colloid Interface Sci. 221, 133 (2000).

${ }^{15}$ S. Simeonov, U. Bass, and A.R. McGurn, Physica B 228, 245 (1996)

${ }^{16}$ X. Wang, X.-G. Zhang, Q. Yu, and B.N. Harmon, Phys. Rev. B 47, 4161 (1993).

${ }^{17}$ A. Moroz, Phys. Rev. B 51, 2068 (1995).

${ }^{18}$ A.R. Williams and J. van W. Morgan, J. Phys. C 7, 37 (1974).

${ }^{19}$ W.H. Butler, A. Gonis, and X.-G. Zhang, Phys. Rev. B 45, 11527 (1992); 48, 2118 (1993).

${ }^{20}$ J.C.M. Garnett, Philos. Trans. R. Soc. London 203, 385 (1904).

${ }^{21}$ H.S. Sözüer, J.W. Haus, and R. Inguva, Phys. Rev. B 45, 13962 (1992).

${ }^{22}$ S.G. Johnson and J.D. Joannopoulos, Opt. Express 8, 173 (2001).

${ }^{23} \mathrm{M}$. Megens (private communication).

${ }^{24}$ P.R. Villeneuve and M. Piché, Phys. Rev. B 46, 4973 (1992).

${ }^{25}$ A. van Blaaderen et al., Manipulating Colloidal Crystallization for Photonic Applications: from Self-organization to do-ityourself Organization, in Ref. 3, pp. 239-251.

${ }^{26}$ F. García-Santamaria, C. López, F. Meseguer, F. López-Tejeira, J. Sánchez-Dehesa, and H.T. Miyazaki, Appl. Phys. Lett. 79, 2309 (2001)
${ }^{27}$ A. van Blaaderen, R. Ruel, and P. Wiltzius, Nature (London) 385, 321 (1997).

${ }^{28}$ A. Moroz, Phys. Rev. Lett. 83, 5274 (1999); Europhys. Lett. 50, 466 (2000).

${ }^{29}$ W.Y. Zhang, X.Y. Lei, Z.L. Wang, D.G. Zheng, W.Y. Tam, C.T. Chan, and P. Sheng, Phys. Rev. Lett. 84, 2853 (2000).

${ }^{30}$ V. Yannopapas, N. Stefanou, and A. Modinos, Comput. Phys. Commun. 113, 49 (1998).

${ }^{31}$ K. Ohtaka, Phys. Rev. B 19, 5057 (1979); J. Phys. C 13, 667 (1980); A. Modinos, Physica A 141, 575 (1987).

${ }^{32}$ Handbook of Optical Constants of Solids, edited by E.D. Palik (Academic, New York, 1985).

${ }^{33}$ M.A. Ordal, R.J. Bell, R.W. Alexander, Jr., L.L. Long, and M.R. Querry, Appl. Opt. 24, 4493 (1983).

${ }^{34}$ I. El-Kady, M.M. Sigalas, R. Biswas, K.M. Ho, and C.M. Soukoulis, Phys. Rev. B 62, 15299 (2000).

${ }^{35}$ K.P. Velikov, A. Moroz, and A. van Blaaderen, Appl. Phys. Lett. 80, 49 (2002).

${ }^{36}$ A. Tip, A. Moroz, and J.-M. Combes, J. Phys. A 33, 6223 (2000).

${ }^{37}$ V. Kuzmiak and A.A. Maradudin, Phys. Rev. B 55, 7427 (1997).

${ }^{38}$ A. Moroz, A. Tip, and J.-M. Combes, Synth. Met. 116, 481 (2001).

${ }^{39}$ S. Fan, P.R. Villeneuve, and J.D. Joannopoulos, Phys. Rev. B 54, 11245 (1996).

${ }^{40}$ V. Yannopapas, A. Modinos, and N. Stefanou, Phys. Rev. B 60, 5359 (1999).

${ }^{41}$ W.H. Zachariasen, Theory of X-ray Diffraction in Crystals (Dover, New York, 1945).

${ }^{42}$ P.S.J. Russel, Phys. World 45(8), 37 (1992).

${ }^{43}$ K.P. Velikov, Ch.G. Christova, R. Dullens, and A. van Blaaderen, Science 296, 106 (2002).

${ }^{44}$ J.P. Hoogenboom, D.L.J. Vossen, C. Faivre-Moskalenko, M. Dogterom, and A. van Blaaderen, Appl. Phys. Lett. 80, 4828 (2002).

${ }^{45}$ P.A. Maia Neto and H.M. Nussenzveig, Europhys. Lett. 50, 702 (2000).

${ }^{46}$ X. Zhang, Z.-Q. Zhang, L.-M. Li, C. Jin, D. Zhang, B. Man, and B. Cheng, Phys. Rev. B 61, 1892 (2000).

${ }^{47}$ A.N. Fang, W.Y. Zhang, Z.L. Wang, A. Hu, and N.B. Ming, J. Phys.: Condens. Matter 13, 8489 (2001).

${ }^{48}$ W.Y. Zhang, Z.L. Wang, A. Hu, and N.B. Ming, J. Phys.: Condens. Matter 12, 9361 (2000).

${ }^{49}$ S.J. Oldenburg, R.D. Averitt, S.L. Westcott, and N.J. Halas, Chem. Phys. Lett. 288, 243 (1998).

${ }^{50}$ A. Moroz and A. Tip, J. Phys.: Condens. Matter 11, 2503 (1999).

${ }^{51}$ W. Kohn and N. Rostoker, Phys. Rev. 94, 1111 (1954).

${ }^{52}$ M. Abramowitch and I.A. Stegun, Handbook of Mathematical Functions (Dover Publications, New York, 1973).

${ }^{53}$ J.S. Faulkner, J. Phys. C 10, 4661 (1977).

${ }^{54}$ B. Segall, Phys. Rev. 105, 108 (1957). 